\title{
Effect of Counterflow Jet on a Supersonic Reentry Capsule
}

\author{
Chau-Lyan Chang ${ }^{*}$ \\ NASA Langley Research Center, Hampton, VA23681 \\ Balaji Shankar Venkatachari ${ }^{\dagger}$ and Gary C. Cheng ${ }^{\ddagger}$ \\ Department of Mechanical Engineering, \\ University of Alabama at Birmingham, Birmingham, AL 35205
}

\begin{abstract}
Recent NASA initiatives for space exploration have reinvigorated research on Apollo-like capsule vehicles. Aerothermodynamic characteristics of these capsule configurations during reentry play a crucial role in the performance and safety of the planetary entry probes and the crew exploration vehicles. At issue are the forebody thermal shield protection and afterbody aeroheating predictions. Due to the lack of flight or wind tunnel measurements at hypersonic speed, design decisions on such vehicles would rely heavily on computational results. Validation of current computational tools against experimental measurement thus becomes one of the most important tasks for general hypersonic research. This paper is focused on time-accurate numerical computations of hypersonic flows over a set of capsule configurations, which employ a counterflow jet to offset the detached bow shock. The accompanying increased shock stand-off distance and modified heat transfer characteristics associated with the counterflow jet may provide guidance for future design of hypersonic reentry capsules. The newly emerged space-time conservation element solution element (CESE) method is used to perform time-accurate, unstructured mesh Navier-Stokes computations for all cases investigated. The results show good agreement between experimental and numerical Schlieren pictures. Surface heat flux and aerodynamic force predictions of the capsule configurations are discussed in detail.
\end{abstract}

\section{Introduction}

Crew rew exploration vehicle (CEV) is one of the important elements in NASA's Constellation Program. This vehicle is intended to replace the aging space shuttle for future human space flights. According to the initial concept development, the CEV consists of two main parts - a conical crew module similar to the Apollo command module and a cylindrical service module which holds the on-board supplies [1]. To build up the knowledge base for future CEV design, aerothermodynamics analysis of the conical capsule thus becomes an important research topic. During the Apollo era, extensive resources were spent at ground [2-3] and flight tests [4-6] of the reentry Command Module $(\mathrm{CM})$ to establish engineering correlations of the surface heating during reentry. Flight tests performed with the Apollo CM capsule at orbital and super-orbital speeds cover a broad regime including both laminar and turbulent flows. The measured afterbody heat transfer data show that theoretical predictions, wind tunnel data can be used to reasonably predict the heating to the conical section of the CM during flight [4]. More recently, Wright et al. performed numerical computations for several Apollo CM reentry cases and the computed afterbody heat flux generally agrees well with the flight data [7].

\footnotetext{
* Research Scientist, Computational Aerosciences Branch, email: Chau-Lyan.Chang@nasa.gov, Senior member, AIAA

${ }^{\dagger}$ Graduate Research Assistant, Dept. of Mechanical Engineering, UAB, email: balaji@uab.edu, Member AIAA.

\# Assistant Professor, Dept. of Mechanical Engineering UAB, email: gcheng@uab.edu, Senior member AIAA.
} 
Effects of the nose cap ablation on the afterbody heating at Mach numbers 5.8 to 8.3 were also investigated [8]. It was found that ablation increases the afterbody pressure but decreases the afterbody heat transfer in the separated flow region and has a negligible effect in the attached flow region. Due to measurement difficulties, the forebody heat transfer data is scarce. Park and Tauber gave an overview of the heatshielding problems during planetary entry [9]. Three flight experimental configurations; the Apollo CM, the Pioneer-Venus, and the Galileo Probe, were investigated theoretically. Heating rate near the stagnation region is compared with the experimental data.

Accurate predictions of the heating rate via either numerical computation or theoretical/experimental correlations are essential for the design of CEV. Overcautious design by applying a large safety factor to cope with possible uncertainties would greatly increase the thermal protection system (TPS) weight. It should be noted that the heating problem is a direct outcome of the flow physics. Many issues including laminar to turbulent transition, radiation heat transfer, surface chemistry, transport property modeling, and surface ablation modeling can affect the accuracy of prediction. The goal of research efforts on the subject is to further our understanding of all these issues via combined experimental and computational investigations for CEV type of configurations.

While more accurate predictions help alleviate design uncertainties, effective flow control may directly impact the heating rate to the vehicle surface. Unfortunately, it is well-known that controlling hypersonic flow is extremely difficult. Active chemical reactions near the hypersonic vehicle surface provide an impetus for possible Magnetofluid dynamic control. However, it is physically unclear how such device can reduce the heating rate. More recently, Daso and Prichett [10] proposed an active flow control concept by altering the leading edge flowfield of the Apollo Command Module like capsules. Control is achieved by applying a counterflow sonic or supersonic jet near the stagnation region of the body. The counterflow can significantly impact the pre-leading edge flow, weaken the shock, and increase the shock stand-off distance. The combined effects may reduce the surface heating. For concept validation, a series of wind tunnel experiments were designed and conducted at NASA Marshall Space Flight Center. The baseline geometry is a 2.6\% scale Apollo capsule as shown in Fig. 1. The tunnel operates at either a Mach number of 3.48 or 4.0. Four different jet mass flow rates were investigated. Schlieren pictures/movies, and heat flux vs. jet mass flow rate are measured by the experiment. The central theme of the present paper is to computationally simulate some of the experimental configurations using a time-accurate Navier-Stokes Computational Fluid Dynamics (CFD) solver. These calculations serve two main purposes: to cross-validate with experimental measurements and to perform more in-depth flow physics investigations of supersonic/hypersonic flow over a capsule.

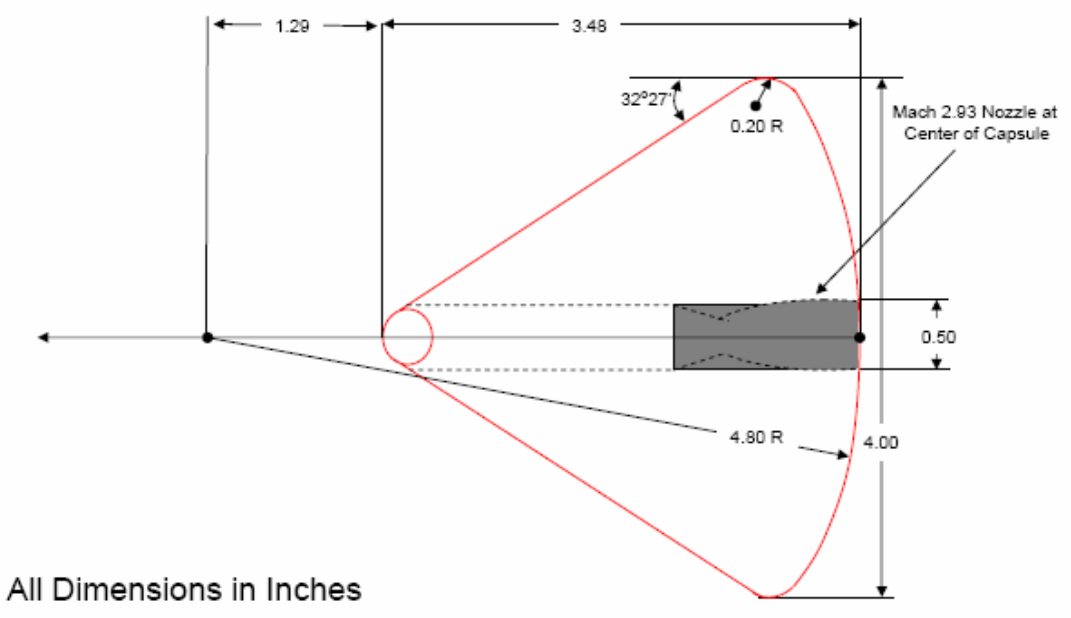

Figure 1: Capsule geometry of a 2.6\% Apollo Command Module with a counterflow nozzle near the centerline of the capsule [10] 
The concept of using counterflow jet to control supersonic flow in fact can be dated back several decades. Following the work of Romeo \& Sterrett [11], Finley [12] did a series of experiments to study the flow physics of counterflow jets on supersonic flows over a hemisphere-cylinder. It was shown that a low-velocity "dead-air" region surrounds the jet in addition to the forward moving of the bow shock. The pressure in the dead-air region is substantially lower than the stagnation pressure of the same body without a counter flow jet. As a result, the total drag force on the body changes significantly in the presence of the counterflow jet. In this way, a counterflow jet works like a structural spike used for drag reduction of a blunt body. Several later works have been carried out along this line of idea (e.g. [13-14]). It was demonstrated that 30-50\% drag reduction can be achieved by counterflow blowing against a supersonic stream of Mach 4 or higher [14]. However, the presence of counterflow jet can also lead to unsteady flow depending on the relative magnitude of the injection and stagnation pressure of the supersonic flow [11-14]. In addition to drag, effect on heat transfer due to the presence of a counterflow jet is another main focus for the present investigation.

The presence of the counterflow jet complicates the grid generation process for CFD calculations. A structured mesh can still be generated by carefully arranging the block grids. However, an unstructured mesh seems to be more suitable for the present configuration topologically. As far as the flow solver is concerned, despite abundant CFD solvers available to-date for hypersonic flow calculations, the present investigation appears to have a more stringent requirement on the solver chosen. When a capsule-like model with a counterflow jet is immersed in a supersonic free-stream, the resulting flowfield is rather "feature rich". The detached bow shock has to be captured well without causing the famous carbuncle phenomenon [15-16]. Interaction of the counterflow jet and the post-shock subsonic stream can lead to a strong unsteady shear layer, which may then feed into the afterbody separation region. Together, strong flow unsteadiness may be present. In addition, the bow shock reflects from the wind tunnel wall and the afterbody shock can lead to a shock-shock interaction. Shocks coming off the shock-shock interaction would also impinge on the sting of the wind tunnel model support and lead to shock boundary layer interaction. Therefore, an unstructured time-accurate Navier-Stokes solver is the most suitable for the present investigation.

Systematic studies of the available numerical methods to search for the best one for the current problem is beyond the scope of this paper. Here, we adopt the emerging space-time conservation element and solution element (CESE) method of Chang [17] for all the computations. A more thorough discussion of the issues related to Navier-Stokes calculations of the CESE method can be found in other works.[18, 19]. The CESE method is constructed by enforcing global and local flux conservations on discretized volumes in the space-time domain. In other words, both temporal and spatial derivatives are treated in the same fashion. Unlike the traditional methods, this approach offers consistent order of accuracy in both time and space and strict flux conservation over the discretized domain. In this new approach, the flux vector integration is carried out on a set of conservation elements. Dependent variables are defined in a set of solution elements surrounding the mesh points. By staggering the conservation elements and solution elements in the space-time domain, no flux reconstruction is necessary at the conservation element interface. The CESE method has been successfully applied to a broad spectrum of flow problems including computational aero-acoustics (CAA), general flow solutions from very low to hypersonic speeds, and electromagnetic wave propagations [20-22]. Many of these results indicate that the CESE method offers high solution accuracy for flows containing both shocks and acoustic or vortical waves. In this paper, we apply the CESE method for the time-accurate viscous calculations of supersonic flows over a set of conical-shape capsule configurations used in the experiment of Daso and Pritchett [10]. The numerical method is described briefly in the next section, followed by detail discussions of all the cases computed. A short summary is drawn at the end.

\section{The Space-Time CESE Method}

Numerical formulation of the space-time CESE method has been discussed in full details in many other papers (e.g. [17-19] and will not be repeated here. Instead, we only give a brief description of the method in this section. The three-dimensional compressible Navier-Stokes equations in conservative form can be written as:

$$
\frac{\partial Q}{\partial t}+\frac{\partial F}{\partial x}+\frac{\partial G}{\partial y}+\frac{\partial H}{\partial z}=I+\frac{\partial F_{v}}{\partial x}+\frac{\partial G_{v}}{\partial y}+\frac{\partial H_{v}}{\partial z}
$$


where the dependent variable vector is defined as $Q=(\rho, \rho u, \rho v, \rho w, e)$ and $x, y, z$, and $t$ represent spatial coordinates and time, respectively. Flow variables $\rho, u, v, w$, and $e$ represent density, three velocity components, and total energy, respectively. Definitions of the inviscid flux vectors $F, G, H$ and viscous flux vectors $F_{v}, G_{v}, H_{v}$ can be found in standard text books and are not included here. The source vector $I$ contains all external forcing or other energy-related source terms. For axisymmetric flows, $H=H_{v}=0$ and $I$ contains source terms associated with the cylindrical coordinate system. Euler equations are recovered by neglecting all viscous terms on the right hand side of the equation. To close the system, the perfect gas relation is used in conjunction with eq. (1).

Unlike conventional CFD algorithms, the space time CESE method is formulated by treating both temporal and spatial directions with synergy. To this end, eq. (1) is re-written in the following integral form

$$
\oint_{S(V)} \vec{h} \cdot d \vec{s}+\int_{\mathrm{v}} I d V=0
$$

where the integration is over the surface $S$ of an arbitrary space-time volume $V$. The flux vector $\vec{h}$ is defined as

$$
\vec{h}=\left(F-F_{v}, G-G_{v}, H-H_{v}, Q\right)
$$

where the surface normal vector is defined by $\vec{s}=\vec{n} d \sigma$, where $d \sigma$ is the area increment on $S$ and $\vec{n}$ is the outward unit normal vector. The CESE method is constructed by performing numerical integrations using Eq. (2) over discretized regions called conservation elements (CE) defined in the space-time volume under consideration. Spatial and temporal coordinates are treated in the same way and no special time integration is required. Before numerical integration can be performed by using eq. (2), the dependent variable vector $Q$ is assumed to vary piecewise linearly within a solution element (SE) according to the following first-order Taylor series expansion,

$$
Q(x, y, z, t)=Q_{0}+Q_{t}\left(t-t_{0}\right)+Q_{x}\left(x-x_{0}\right)+Q_{y}\left(y-y_{0}\right)+Q_{z}\left(z-z_{0}\right)
$$

where $Q_{0}$ is the solution vector at the solution point $\left(x_{0}, y_{0}, z_{0}, t_{0}\right)$ defined as the geometry center of the polygons formed by all surrounding conservation elements. First order derivatives $Q_{v}, Q_{x}, Q_{y}$, and $Q_{z}$.are constants over the solution elements. The time derivative, $Q_{t}$, can be expressed in terms of spatial derivatives by using the discretized form of the governing partial differential equations. Note that both the solution vector and derivatives are treated as unknowns in the CESE method.

Given one more degree of freedom in time, there are many ways to integrate eq. (2) over the domain of interest. For simplicity, the conservation elements are constructed by an element interface parallel to the temporal axis. In theory, the conservation elements can take any arbitrary shape in the space-time domain. However, this would require an extra dimension (in time) for the mesh generation process. Furthermore, the resulting discretized equations for the unknowns at the new time level would involve a larger stencil and thus more computational time. The CESE method is constructed with a staggered conservation element and solution element, flow variables and flux vectors are uniquely defined at all the conservation element interfaces. Thus, no Riemann solver is necessary. By numerically integrating the flux conservation equations (2) over all surrounding conservation elements, we have a set of coupled algebraic equations for $Q_{0}$. If the solution point is selected as the geometrical center of all surrounding CE, the sum of these algebraic equations contains only solution vectors and derivatives at the old time level in addition to the unknown $Q_{0}$ and can be easily solved. In the a-scheme, the remaining unknown derivatives $Q_{x}, Q_{y}$, and $Q_{z}$ can be solved by using the three algebraic conservation equations. The a-scheme gives a dissipation free solution and artificial numerical dissipation must be added to maintain numerical stability for nonlinear equations. This is done by computing derivatives using a weighted average by accounting for variations among all neighboring solution elements. A special case referred to as the c-scheme is formulated by using only the weighted average for all derivatives. The resulting scheme is numerically stable if the CFL number is less than unity. It should be noted that numerical dissipation is a function of CFL number; smaller values causes more numerical dissipation. In a later paper by Chang [19], a CFL-insensitive scheme is formulated by moving the surrounding points used in the weighted average derivatives calculations. With this new approach, numerical dissipation can be controlled for small CFL numbers. 
Boundary conditions are implemented via a simple flux balance at the boundary face. Implementation of a nonreflected boundary condition is relatively easy for the CESE method. In conventional CFD methods, characteristic variables (Riemann invariants) in the direction normal to the boundary are used to manually limit the traveling directions of characteristic information. For multi-dimensional problems, this one-dimensional characteristic splitting is not exact and introduces errors that cause boundary reflections. A buffered domain or sponge layer near the boundary is usually introduced to further alleviate boundary reflections. In contrast, a simple steady-state extrapolation boundary condition without a buffered domain works well for the CE/SE method. This nice property is associated with the conservation of flux along the temporal direction. Global flux formulation along all directions allows information to propagate in time and exit the domain without incurring significant boundary reflections. However, for very small-amplitude perturbations, a limited extent of reflection at the boundary may present. However, it can be easily eliminated by a relatively small buffered domain.

When applied to low Mach number flows, the CESE method can preserve the solution accuracy without any preconditioning of the governing equations. Solving low speed flows using the compressible governing equations suffers from error accumulation originating from the disparity in the eigenvalues of the hyperbolic system. Modifications of the equations to alter the propagation speed of the characteristic variables are often required to improve convergence rate and solution accuracy. It has been shown that the CESE method can provide highly accurate solutions even after long time integration without any preconditioning techniques. The convergence rate remains slow because of the presence of disparate eigenvalues, but the error accumulation problem does not exist for the CESE method.

Numerical formulations of the CESE method for the Euler equations are directly applicable to the Navier-Stokes equations provided that several important issues are properly addressed. Firstly, viscous terms must be added to the flux vectors. These terms involve derivatives of dependent variables. Within the framework of the CESE method, these derivatives are assumed to be constant over the solution element. To account for variations of derivatives within each solution element, higher derivatives must be introduced and evaluated. The second issue related to viscous calculations is the near wall grid stretching. Without proper grid clustering near the solid wall boundary, effects of viscosity cannot be properly accounted for. High aspect ratio grid near the solid wall may slow down the convergence rate and significantly affect the robustness of the solver. For steady-state problems, a constant CFL number (local time-stepping) is usually used to accelerate convergence. For unsteady calculations, a constant time step must be used for all elements, the local CFL number thus varies from 1 (to maintain numerical stability) near the wall to a very small value in the coarse mesh region. A smaller CFL number implies a larger numerical dissipation for the $c$-scheme [17]. The disparity in CFL number thus may result in adverse effects on the solution accuracy. To control numerical dissipation, the CFL number insensitive method [23] must be used. The third issue is related to the viscous wall boundary conditions. No slip velocities and thermal boundary conditions are enforced in a ghost cell adjacent to the solid wall. Detailed discussion of this issue can be found in [19]. The CESE Navier-Stokes code, $e z 4 d$, developed at NASA Langley [19] is used for all the calculations in this study.

\section{Results and Discussion}

The $e z 4 d$ code has been validated for both steady and time-accurate compressible viscous flows [19]. Time accuracy is essential for the present investigation because of the intrinsically unsteady nature of the flowfield around a capsule-like geometry. Computational studies of the experimental configurations of Daso and Pritchett [10] is the central theme of this paper. For a zero degree angle of attack, the axisymmetric version of the Navier-Stokes equations can be used for calculation. Only half of the domain is calculated due to symmetry. The triangular mesh for the baseline configuration without any counterflow jet is shown in Fig. 2. There are a total of about 32,000 nodes and 63,000 elements. Although systematic grid convergence study was not performed, we calculated the flow around the baseline configuration using two different meshes. A more refined mesh with 188,000 nodes and 372,000 elements as shown in Fig. 3 was also used for comparison. The overall mesh is finer and in addition, clustering meshes are generated both near the wind tunnel and model walls to resolve the boundary layer. The wind tunnel flow conditions used correspond to Case 1 to be described below. Figure 4 compares the computed Mach number contours using these two grids. Note that the physical times of these two snap shots are not identical. Nevertheless, the overall computed flowfield features are similar. The leading edge shock caused by the growth of the wind tunnel boundary layer is better resolved in the fine mesh calculation but it does not have any major effect on the flow 
development downstream. The overall agreement near the cone region is quite good although there are visible differences after the shock-shock interaction located around about half of the sting length. Despite all these downstream discrepancies, the flowfield features around the capsule is well resolved by the coarse mesh. Since we are more concerned with the heat transfer phenomena near the capsule face, the coarse mesh is used for all calculations presented below.

There are a total of 8 cases performed for zero angle of attack based on the experimental setup. Table 1 summarizes the free-stream Mach numbers for these eight cases along with the computed overall force and heat transfer responses. These eight conditions belong to two groups of wind tunnel conditions. The first group, corresponds to Cases 1 through 4, is with a free-stream Mach number of 3.48, stagnation temperature of $333 \mathrm{~K}$, and stagnation pressure of $3.061 \mathrm{~atm}$. The second wind tunnel conditions, correspond to Cases 5-8, are at a Mach number of 4.96, stagnation temperature of $333 \mathrm{~K}$, and stagnation pressure of $5.442 \mathrm{~atm}$. The resulting unit Reynolds number for the two wind tunnel conditions are $1.527 \times 10^{7} \mathrm{~m}^{-1}$ and $1.365 \times 10^{7} \mathrm{~m}^{-1}$, respectively. The unit Reynolds number behind the bow shock is around 1.1 million per foot. For a model with a base diameter of about 4 inch, the resulting Reynolds number on the capsule face is rather small; therefore, laminar flow is assumed for all calculations presented herein. However, turbulent flow may still be present at the conical section or sting of the wind tunnel model. Boundary conditions are as follows: symmetry at the centerline of the wind tunnel; free stream and nonreflecting conditions at the inlet and exit, respectively; inviscid wall at the wind tunnel wall, and no-slip conditions on the capsule and sting. The viscous effect on the wind tunnel wall is unimportant and neglected. The wall temperature of the capsule is set at $297 \mathrm{~K}$. Table 1 also lists the quasi-steady state maximum and minimum heat fluxes at the capsule face. The locations where these peak heating or cooling occur vary from configuration to configuration. Three different counterflow jet conditions at Mach numbers of 1.0, 2.44, and 2.94 are incorporated in the capsule as summarized in Table 2 .

Before discussing in full detail all the cases, we verify the computational results by comparing them with the experimental flow visualization. Figure 5 shows the experimental and numerically generated Schlieren pictures for the baseline (no jet) Case 1. The shock stand-off distance and other major flow features are captured very well by numerical calculations. Figure 6 compares the results for Case 3 with a Mach 2.44 counterflow jet against experimentally taken Schlieren photo for the same jet Mach number but with a smaller mass flow rate. Again, all the major features associated with the jet are captured very well in the computations. The difference in the jet plume size is probably due to a larger jet mass flow rate used in the computation. In general, the numerical Schlieren pictures are sharper and contain more flow details due to higher resolution used. It remains to be verified that these additional details are relevant. Nonetheless, these results serve to verify the computational results in this study.

Drag is one of the most import design parameters for a reentry capsule. For all cases, we calculate the total drag of the configuration and monitor its transient response. In addition to the pressure and viscous drag on the capsule surface, the momentum due to mass injection into the free stream from the control jet also has a significant contribution to the total drag. For an unstructured mesh, it is non-trivial to integrate the total momentum at the jet exit. Instead, we first compute the viscous and pressure drag on the nozzle wall of the jet. From this nozzle wall drag, along with the known nozzle inlet mass flow rate and momentum, the nozzle exit momentum can be deduced easily. In addition to total drag, heat transfer is also monitored during all transient calculations. The average heat flux, along with maximum and minimum heat flux on the capsule face is calculated at all physical time being computed. The average heat flux here is defined as the overall heat transfer to the capsule divided by the total (capsule only) surface area. For each case under investigation, we present flowfield visualization, drag, and the heat flux characteristics computed. The goal is to document all cases to aid future design consideration. Comparisons with experimental data may also be performed when it becomes available.

The same cases were also computed by using a pressure-based Reynolds Averaged Navier-Stokes code [24-25]. Instead of using the axisymmetric formulation, a full 3D mesh was used for all these calculations. In addition, the standard k-epsilon model is used for these 3D RANS computations. The results indicate a very mild 3D variation. The predicted drag is compared with those obtained in this study in Table 3. For the baseline configurations (Cases 1 and 5), the difference is about $3 \%$ and $6 \%$, respectively. The cases with a counterflow jet differ more. However, both sets of calculations show that drag reduces when there is a counterflow jet for the Mach 3.48 free-stream conditions. This trend reverses (drag increases) for the Mach 4.98 cases. We will discuss this phenomenon in more detail below. The reason for the discrepancies in predicted drag between these two codes, on top of different meshes, 
is partly due to the differences in laminar and turbulent flow assumptions. One other possible cause can be attributed to the difference in calculating the drag. In 3-D calculations, the friction loss of the chamber wall and the support sting was not included. Despite some differences in the computed drag, the overall agreement of the computed capsule surface pressure as shown in Fig. 7 is good. The small hump of the pressure distribution near the center line from the 3D RANS results is probably associated with a very dense mesh used near the center of the capsule face. This dense mesh is mainly due to topological requirements of the block structured mesh generation, not by physical considerations. For the cases with jet, the results are more sensitive to the grid distribution due to their unsteady nature. As a result, more notable differences are observed. More studies are necessary to clarify this issue.

We first present the baseline case without any counterflow jet. Figure 8 shows the contours of calculated pressure, Mach number and temperature for Case 1 . The time history of predicted drag (421.8 N), maximum (1160 \pm 160 $\left.\mathrm{W} / \mathrm{m}^{2}\right)$, minimum $\left(-425 \mathrm{~W} / \mathrm{m}^{2}\right)$, and averaged $\left(-75 \pm 15 \mathrm{~W} / \mathrm{m}^{2}\right)$ heat fluxes exerted on the capsule face are plotted in Figures 9 and 10, respectively. Negative heat flux means cooling. The fluctuation levels given hereafter for the computed heat flux and drag represent the overall maximum and minimum values of all physical time (including initial transients). The quasi-steady state values of the maximum and minimum heat fluxes are listed in Table 1 . The computed drag force on the capsule indicates a quick convergence to steady-state. However, some small scale unsteadiness is still visible in the flowfield. All the important flow features such as the bow shock, reflected shock, shock-boundary layer interaction, and shock-shock interaction are captured well in the simulation. Note the sizable separation zone in the conical section of the capsule. The shock coming off the end of the conical section interacts with that reflected form the wind tunnel wall, forming a shock-shock intersection point. The tunnel wall reflected shock further hits the sting wall and causes shock-boundary layer interaction. This interaction triggers a substantial flow separation. More grid refinement studies are necessary to make sure that it has adequate grid resolution in the region. Because of a cold capsule wall $(297 \mathrm{~K})$, the model surface ends up being cooled by the post shock stream (negative average heat flux). The peak heating occurs around the shoulder of the capsule (i.e. intersection of the face and conical section) while the minimum heat flux point is located at about 1.5 inch from the centerline.

Case 2 is with a counterflow jet exit Mach number of 1 . The computed pressure, Mach number and temperature contours are shown in Figure 11. The time history of the calculated drag force $(361 \pm 1.5 \mathrm{~N})$ of Case 2 is plotted in Figure 12, along with that of Case 1 for comparison. It can be seen that the interfaces of the shock for Case 2 are not as distinct as those with no counterflow jet probably because the strength of the shock is reduced by the counterflow jet. The shock stand-off distance is increased by a factor of about 4 to 5 . Compared with Case 1 without a jet, some flow unsteadiness is evident due to the small-scale oscillations in the drag history. As a result of the counterflow jet, the total drag is reduced by about $14 \%$. Figure 13 exhibits the time history of the maximum $\left(8400 \mathrm{~W} / \mathrm{m}^{2}\right)$, minimum

Table 1: Summary of the numerical results for the simulations completed

\begin{tabular}{|c|c|c|c|c|}
\hline & & Drag force $(\mathrm{N})$ & $\%$ Change in drag & Heat flux $\left(\mathrm{W} / \mathrm{m}^{2}\right)$ \\
\hline \multirow{4}{*}{$\begin{array}{c}\mathrm{M}_{\infty}=3.48 \\
\alpha=0 \\
\mathrm{P}_{\mathrm{t}}=3.061 \mathrm{~atm}\end{array}$} & $\mathrm{M}_{\mathrm{j}}=0($ Case 1$)$ & 421.8 & 0 & $-350 \sim 410$ \\
\hline & $\mathrm{M}_{\mathrm{j}}=1($ Case 2$)$ & $361 \pm 1.5$ & $-14.3 \%$ & $-400 \sim 8400$ \\
\hline & $\mathrm{M}_{\mathrm{j}}=2.44($ Case 3$)$ & $321 \pm 8$ & $-23.8 \%$ & $-2500 \sim 7800$ \\
\hline & $\mathrm{M}_{\mathrm{j}}=2.94($ Case 4$)$ & $242 \pm 12$ & $-42.5 \%$ & $-2600 \sim 3000$ \\
\hline \multirow{4}{*}{$\begin{array}{c}\mathrm{M}_{\infty}=4.96 \\
\alpha=0 \\
\mathrm{P}_{\mathrm{t}}=5.442 \mathrm{~atm}\end{array}$} & $\mathrm{M}_{\mathrm{j}}=0($ Case 5$)$ & 213.5 & 0 & $-340 \sim 500$ \\
\hline & $\mathrm{M}_{\mathrm{j}}=1($ Case 6$)$ & $390 \pm 15$ & $+82.7 \%$ & $-800 \sim 5500$ \\
\hline & $\mathrm{M}_{\mathrm{j}}=2.44($ Case 7$)$ & $326.5 \pm 2$ & $+52.9 \%$ & $-100 \sim 5300$ \\
\hline & $\mathrm{M}_{\mathrm{j}}=2.94($ Case 8$)$ & $380 \pm 55$ & $+78 \% 0$ & $-425 \sim 5200$ \\
\hline
\end{tabular}


Table 2: Summary of three counterflow jet conditions

\begin{tabular}{|c|c|c|c|}
\hline Exit Mach Number & Stagnation Pressure (atm) & Stagnation Temp. (K) & Mass Flow Rate $(\mathrm{kg} / \mathrm{sec})$ \\
\hline 1.0 & 15.17 & 300 & 0.4536 \\
\hline 2.442 & 37.925 & 300 & 0.4536 \\
\hline 2.937 & 60.68 & 300 & 0.4536 \\
\hline
\end{tabular}

Table 3: Comparison of computed drag with other 3D Navier-Stokes code [24-25]

\begin{tabular}{|c|c|c|c|}
\hline \multicolumn{2}{|c|}{} & Drag force (CESE) & Drag force (3D N-S code [24-25]) \\
\hline \multirow{4}{*}{$\begin{array}{c}\mathrm{M}_{\infty}=3.48 \\
\alpha=0 \\
\mathrm{P}_{\mathrm{t}}=3.061 \mathrm{~atm}\end{array}$} & $\mathrm{M}_{\mathrm{j}}=0$ (Case 1) & $421.8 \mathrm{~N}$ & $434.4 \mathrm{~N}$ \\
\cline { 2 - 4 } & $\mathrm{M}_{\mathrm{j}}=1$ (Case 2) & $361 \pm 1.5 \mathrm{~N}$ & $292 \pm 8 \mathrm{~N}$ \\
\cline { 2 - 4 } & $\mathrm{M}_{\mathrm{j}}=2.44$ (Case 3) & $321 \pm 8 \mathrm{~N}$ & $330 \pm 16 \mathrm{~N}$ \\
\cline { 2 - 4 } & $\mathrm{M}_{\mathrm{j}}=2.94($ Case 4$)$ & $242 \pm 12 \mathrm{~N}$ & $334 \pm 26 \mathrm{~N}$ \\
\hline \multirow{3}{*}{$\begin{array}{c}\mathrm{M}_{\infty}=4.96 \\
\alpha=0\end{array}$} & $\mathrm{M}_{\mathrm{j}}=0($ Case 5) & $213.5 \mathrm{~N}$ & $226.8 \sim 227.8 \mathrm{~N}$ \\
\cline { 2 - 4 } $\mathrm{P}_{\mathrm{t}}=5.442$ atm & $\mathrm{M}_{\mathrm{j}}=1$ (Case 6) & $390 \pm 15 \mathrm{~N}$ & $267.8 \pm 4 \mathrm{~N}$ \\
\cline { 2 - 4 } & $\mathrm{M}_{\mathrm{j}}=2.44$ (Case 7) & $326.5 \pm 2 \mathrm{~N}$ & $312 \pm 19 \mathrm{~N}$ \\
\cline { 2 - 4 } & $\mathrm{M}_{\mathrm{j}}=2.94$ (Case 8) & $380 \pm 55$ & $315 \pm 6$ \\
\hline
\end{tabular}

$\left(-120 \pm 440 \mathrm{~W} / \mathrm{m}^{2}\right)$, and averaged $\left(230 \pm 150 \mathrm{~W} / \mathrm{m}^{2}\right)$ values of the calculated heat flux, all of them also reveal flow unsteadiness. In addition, the averaged heat flux is higher than that with no counterflow jet. The average flux now is positive, indicating heating of the capsule. The peak heat flux for Case 2 is substantially higher than that of Case 1, probably due to the presence of a large low speed region between the bow shock and the capsule face. It is interesting to note that maximum heat flux now occurs near the lip of the counterflow jet. In fact, for all three counterflow jet Mach numbers, the peak heating is always located around the jet nozzle lip. On the other hand, the minimum heat flux point fluctuates about midway between the shoulder of the capsule and the nozzle lip due to the flow unsteadiness.

Computational results of Case 3 with a counterflow jet exit Mach number of 2.44 are depicted in Fig. 14 by showing pressure, Mach number, and temperature contours. The corresponding time history of the predicted drag ( $321 \pm 8 \mathrm{~N})$, maximum $\left(7800 \mathrm{~W} / \mathrm{m}^{2}\right)$, minimum $\left(-2500 \pm 3500 \mathrm{~W} / \mathrm{m}^{2}\right)$, and averaged $\left(800 \pm 1800 \mathrm{~W} / \mathrm{m}^{2}\right)$ heat fluxes exerted on the capsule face are plotted in Figures 15 and 16, respectively. The larger jet exit speed results in a slightly smaller plume angle (between the boundary of the high speed jet plume and the centerline) as compared to Case 2. A shear layer coming off the tip of the jet plume is clearly visible. The drag and heat flux time histories clearly exhibit a lowfrequency, large-amplitude oscillations. These oscillations are probably related to the large-scale structures caused by flow instability of the tip shear layer. The drag force is further reduced ( $24 \%$ smaller than that of Case 1$)$. Meanwhile, the averaged heat flux of Case 3 is higher than that of Case 1 and Case 2. However, the peak heating is about the same as that in Case 2.

Figure 17 shows the calculated flowfield of Case 4 with a counterflow jet exit Mach number of 2.94. The bow shock is pushed further upstream. The jet plume tip shear layer is still present. However, this shear layer appears to impinge on the intersection of the capsule face and conical section and creates a second shear layer. For all three cases with counterflow jet, the remaining shocks behind the first bow shock appear to be much weaker than Case 1 . In particular, for case 4, these afterbody shocks are almost invisible due to the weakened bow shock upstream. The 
separation region, starting from the conical section, appears to be much larger. The time history of the predicted drag $(242 \pm 12 \mathrm{~N})$ of this case is shown in Figure 18. It can be seen that the time averaged drag force is reduced by about $42.5 \%$ as compared to Case 1 . At the end of the simulation, the average drag does not seem to asymptote to a quasisteady state value but the rate of increase has decreased significantly. Figure 19 demonstrates the time history of the predicted maximum $\left(3150 \pm 1350 \mathrm{~W} / \mathrm{m}^{2}\right)$, minimum $\left(-2600 \pm 2600 \mathrm{~W} / \mathrm{m}^{2}\right)$, and averaged $\left(1000 \pm 1000 \mathrm{~W} / \mathrm{m}^{2}\right)$ heat fluxes exerted on the capsule face. The averaged heat flux is the highest among all four cases with a Mach number of 3.48. The amplitude of unsteady oscillations in the drag and heat transfer also is the highest. It appears that the high velocity of the control jet now has an overwhelming effect on the wind tunnel stream. Large scale instability waves off the shear layer caused by the jet plume are playing a dominant role for this case.

In summary for the first wind tunnel conditions, the counterflow jet significantly increases the shock stand-off distance. The higher the jet Mach number, the longer the stand-off distance is. The jet plume also works as a displaced body of the capsule. The very blunt leading edge of the capsule becomes significantly less so; and as a result, the drag reduces. The drag reduction is about $15-43 \%$ for three different jet Mach numbers. The amount of drag reduction seems to be in line with that observed for a blunt hemisphere-cylinder [14]. The presence of the counterflow jet also results in an increase in the average wall heat flux. In general, the peak and valley (negative) heat fluxes become much larger when there is a counterflow jet (see Table 1). This implies that the temperature in the "dead" zone between the boundary of the jet plume and the capsule face varies a lot in the presence of a jet. Evidently, at the current model wall temperature, no reduced surface heating is observed. The actual benefit on heat transfer to the capsule thus cannot be properly assessed in this experimental configuration.

We now turn to the last four cases with a wind tunnel Mach number of about 5. The computed pressure, Mach number and temperature contours for Case 5 are shown in Figure 20. The time history of predicted drag (213.5 N), maximum $\left(503 \mathrm{~W} / \mathrm{m}^{2}\right)$, minimum $\left(-340 \mathrm{~W} / \mathrm{m}^{2}\right)$, and averaged $\left(-103 \mathrm{~W} / \mathrm{m}^{2}\right)$ heat fluxes exerted on the capsule face are plotted in Figures 21 and 22, respectively. These result shows that the flow reaches steady-state very quickly. Moreover, the drag force and heat fluxes are smaller than that of Case 1. The decrease in the drag force is about proportional to the ratio of total wind tunnel momentum (free stream density multiply by free-stream velocity square) between these two cases. The shock stand-off distance at this higher free-stream Mach number is only slightly smaller than that for Case 1. Calculated heat flux is also slightly smaller but comparable to that for Case 1 because the post-shock static temperature is about the same for both conditions according to the 1D normal shock table.

The computed flowfield, drag, and heat flux time history in the presence of three counterflow jet Mach numbers, 1 , 2.44, and 2.94, are shown in Figures. 23-31. The ratio of jet stagnation pressure to the free-stream Pitot pressure (stagnation pressure after the normal shock) for these three cases is about twice the values of the corresponding Cases 2-4 (free stream Mach no. of 3.48) mainly because of the higher free stream Mach number and stronger normal shock. This ratio is usually used as an indicator of the strength of the counterflow jet [12]. A stronger jet implies more significant effect on the supersonic free stream. This is indeed evident from the fact that the shock stand-off distance is relatively longer as compared to Cases 2-4. For Case 8, the bow shock is in fact located quite close to the wind tunnel entrance (Fig. 23). The extent that the jet plume travels upstream is also larger for these three cases. As a matter of fact, the sizes of the plumes are either the same or bigger than the capsule itself (Figures 17,20 , and 23). 

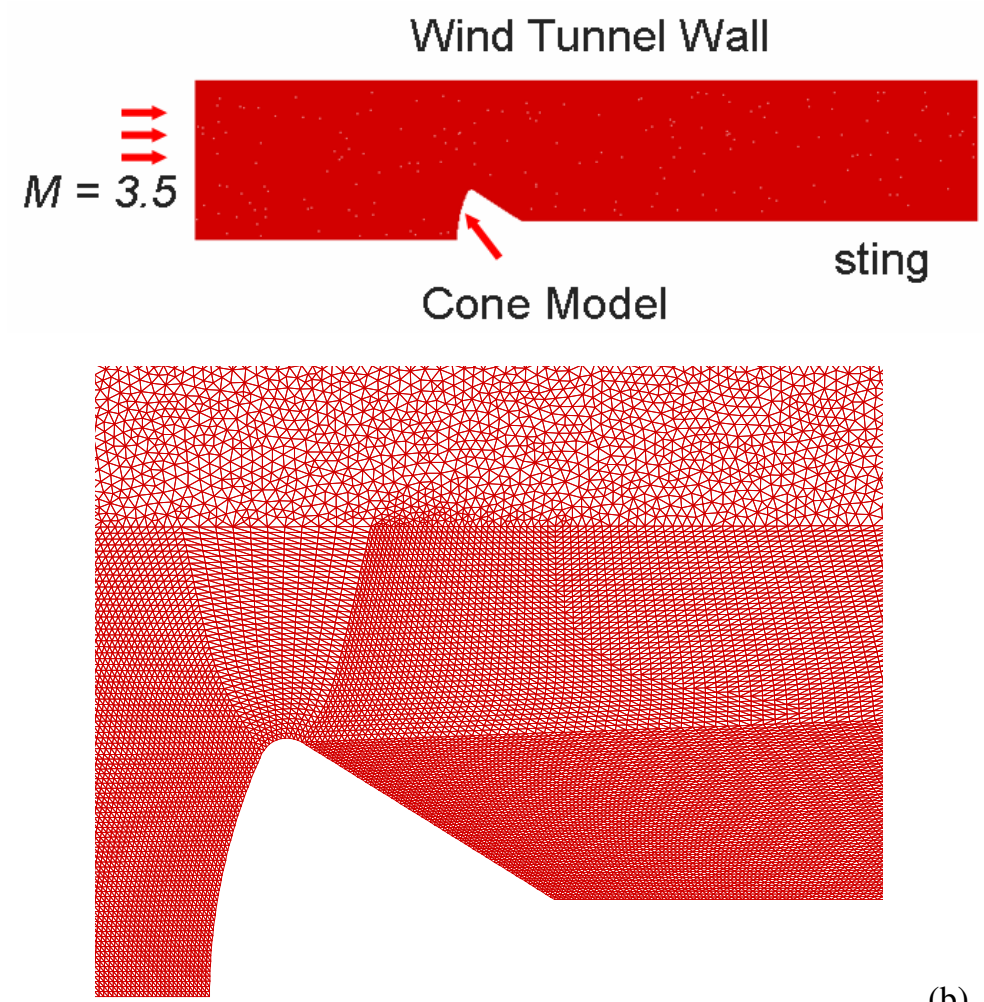

(a)

(b)

Figure 2: Triangular mesh for the baseline capsule configuration including the model and sting (a) mesh, (b) enlarged view near the capsule

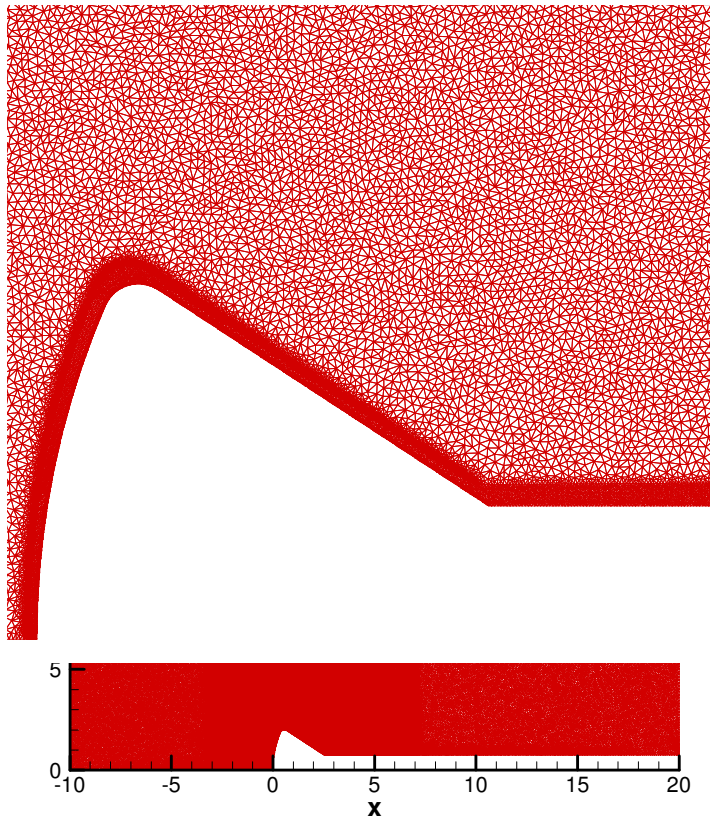

Figure 3: Refined triangular mesh for the baseline configurations and its close-up view

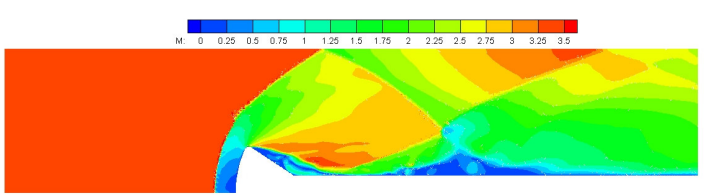

(a)

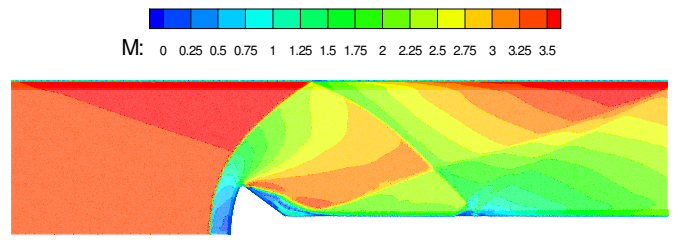

(b)

Figure 4: Comparison of Mach number contours for two meshes (a) coarse mesh (b) fine mesh 


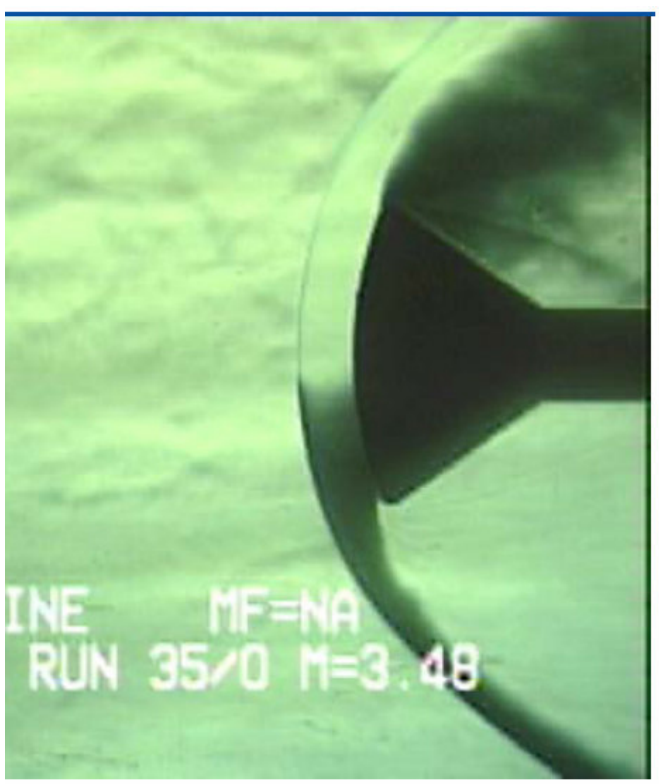

(a)

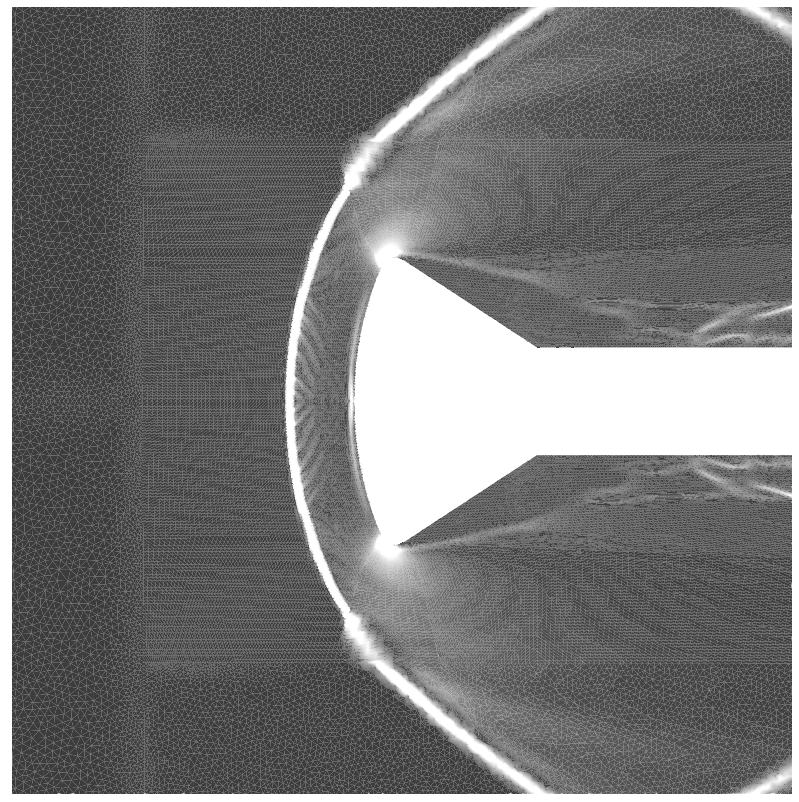

(b)

Figure 5: Comparison of computational and experimental results for the baseline (no jet) configuration: (a) experimental Schlieren picture, (b) numerical Schlieren

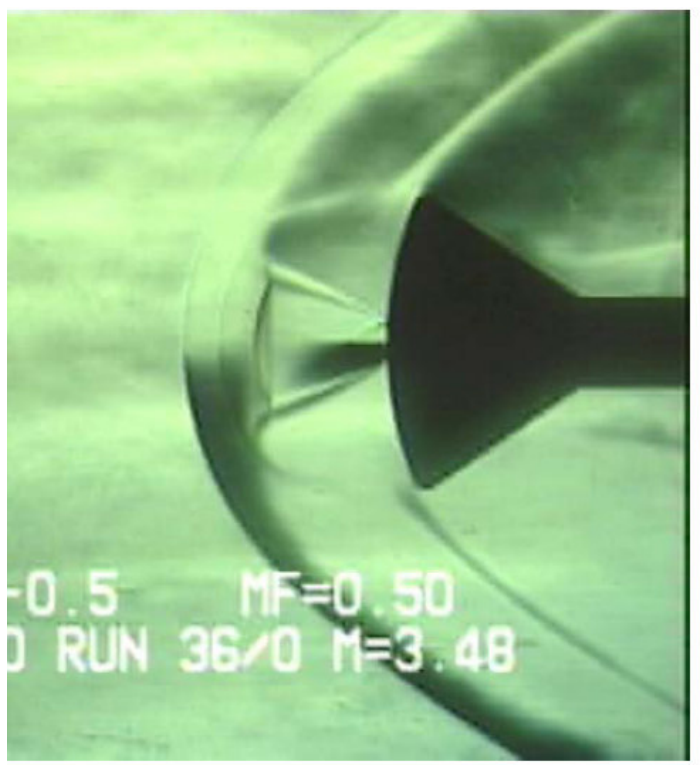

(a)

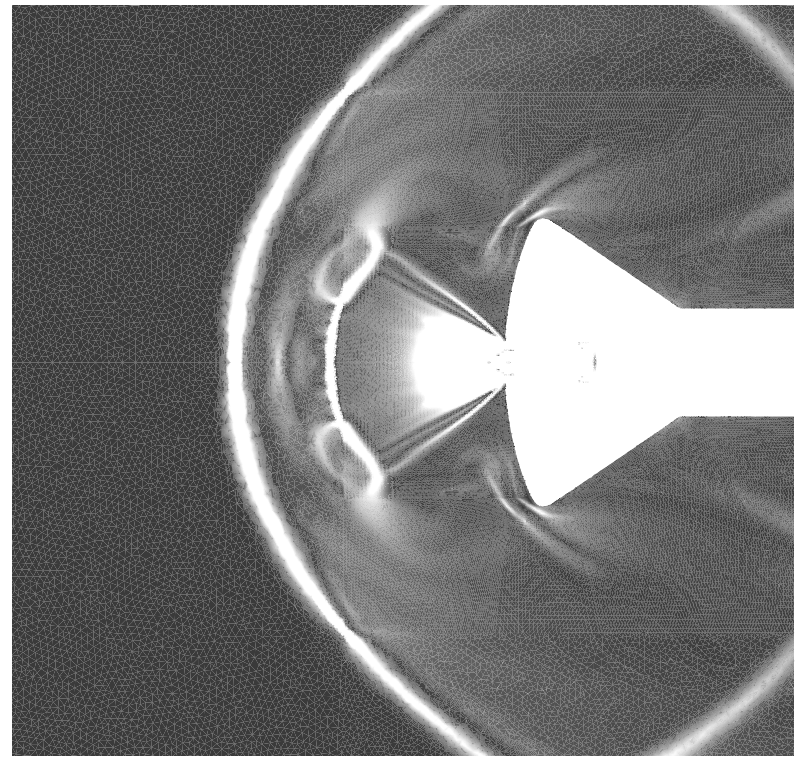

(b)

Figure 6: Comparison of computational and experimental results for Case 3 configuration: (a) experimental Schlieren picture, (b) numerical Schlieren 
The time history of the calculated drag force $(390 \pm 15 \mathrm{~N})$ of Case 6 (Fig. 18) is about $83 \%$ higher than that of Case 5 (no jet). The predicted drag for Case 7 and Case 8 are $(326.5 \pm 2 \mathrm{~N})$ and $(380 \pm 55 \mathrm{~N})$, respectively. These values correspond to about 53\% and 78\% higher than Case 5. Unlike the previous Mach 3.5 cases, the total drag actually increases for all three jet Mach numbers. Results obtained by using the other 3D code [24-25] also show similar drag increase (see Table 3). One possible explanation is that in contrast to the Mach 3.5 cases where the presence of jet makes the effective body (one that is displaced by the jet plume) more slender, the displaced body is effectively more blunt and thus results in a higher drag than the capsule alone. It is unclear why the computed drag does not increase monotonically with the jet Mach number (Case 6 has the largest drag). Calculations with an unbounded free stream without wind tunnel walls may help clarify this issue.

The (absolute) average heat flux on the capsule face of Cases 6-8 is both higher than the baseline Case 5 but the value again does not increase monotonically with the jet Mach number. Among the three cases, only Case 7 converges to an almost steady-state value. Strong unsteadiness is present for the other two cases. From the drag and heat flux plots, it is evident that the flow is unsteady and there exists a dominant frequency for Case 6 and Case 8 . It is again not clear why Case 7 converges to a steady state but not the other two cases. Contour and time history plots for Cases 6 and 8 indicate presence of a dominant frequency. More transient data analysis may help understand the underlying physical mechanisms.

By and large, the four cases computed for the second set of wind tunnel conditions show more dramatic effects of the counterflow jet. Unlike the previous four cases, the total drag is enhanced. Due to the cold wall temperature, it is more difficult to discern the effect on heat transfer to the capsule face. Both peak heating and cooling rate in general increase due to the presence of counterflow jet (see Table 1). Qualitatively, the second set of results is consistent with those for the smaller free-stream Mach number. Full 3D calculations for the same model at 10 degree angle of attack are currently underway. It remains to be seen how three-dimensionality alters the unsteady nature of the flow.

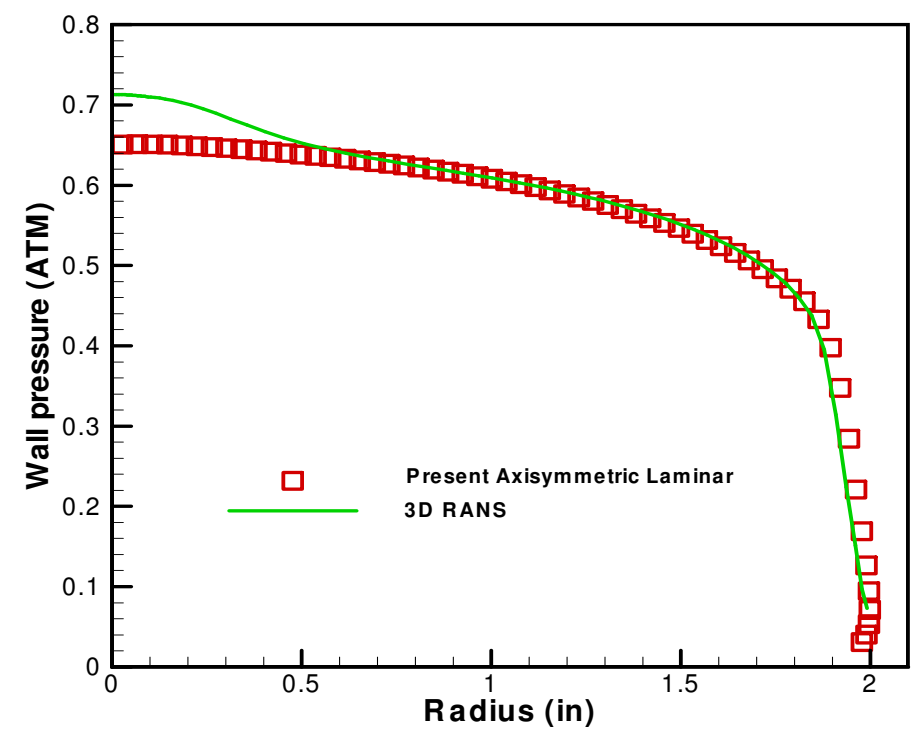

Figure 7: Comparison of computed capsule surface pressure distributions for the present calculations and the 3D RANS results [24-25] for the baseline (no jet) configuration. 


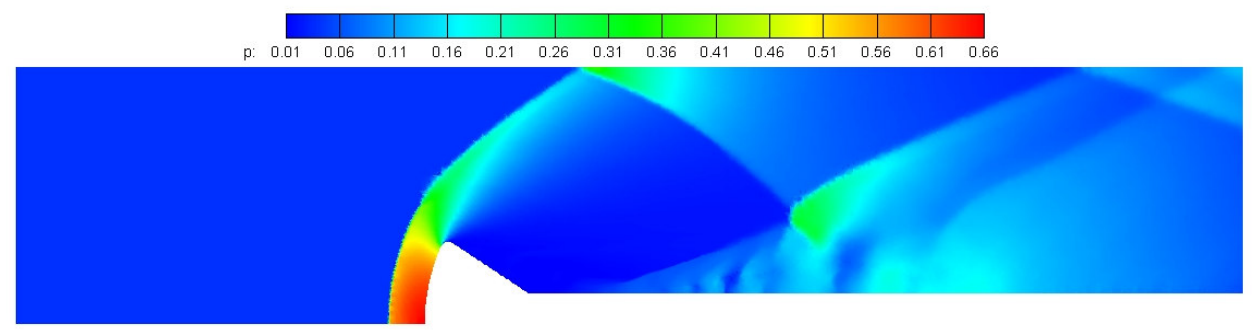

(a) Pressure (atm)

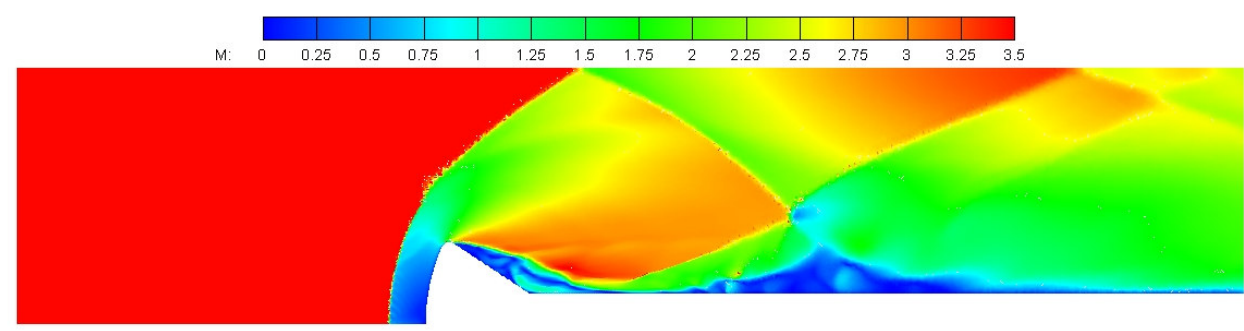

(b) Mach number

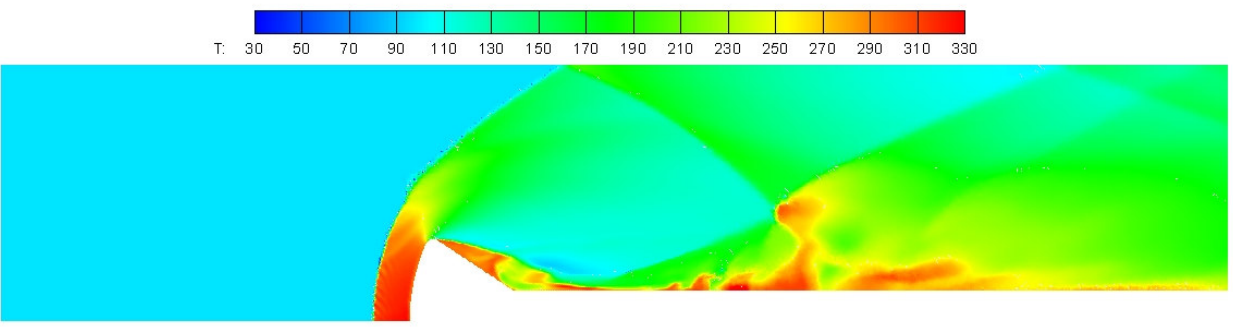

(c) Temperature (K)

Figure 8: Computed flowfield at the symmetry plane for Case $1(\mathrm{M}=3.48$, no jet).

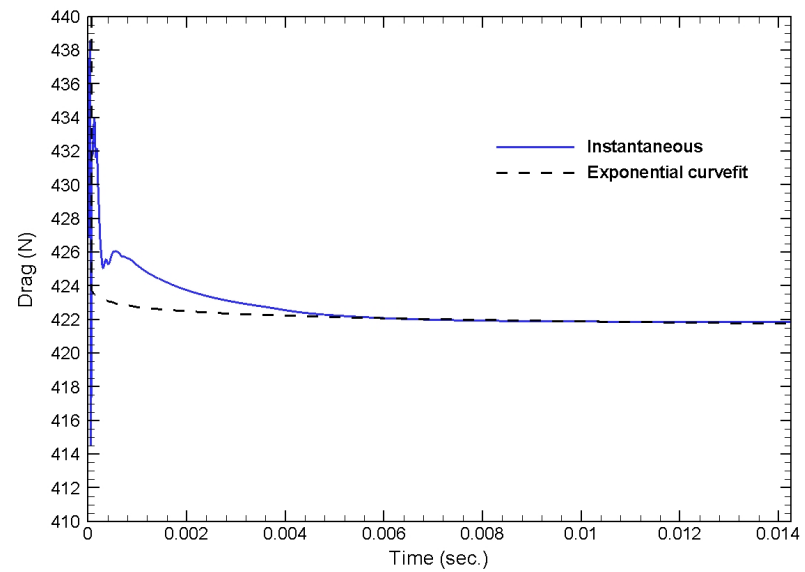

Figure 9: Time history of the drag force on the face of the capsule for Case $1(\mathrm{M}=3.48$, no jet $)$.

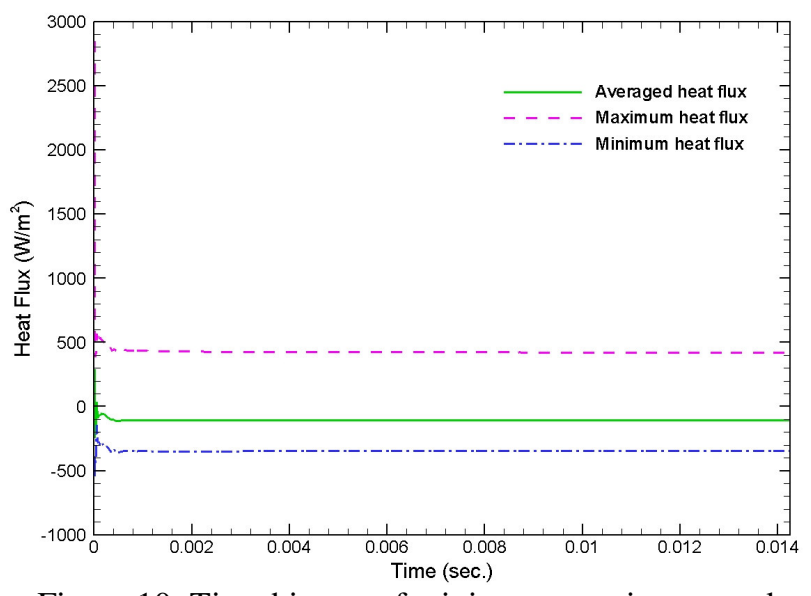

Figure 10: Time history of minimum, maximum, and averaged heat fluxes to the capsule face for Case $1(\mathrm{M}=$ 3.48 , no jet). 


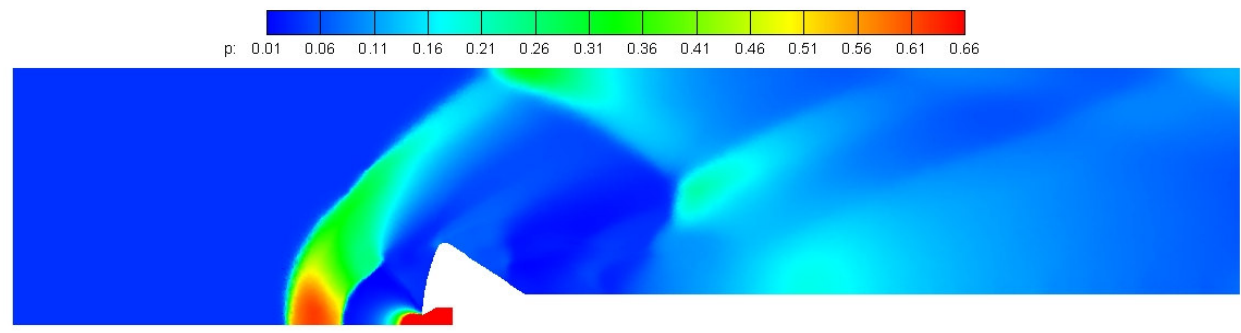

(a) Pressure (atm)

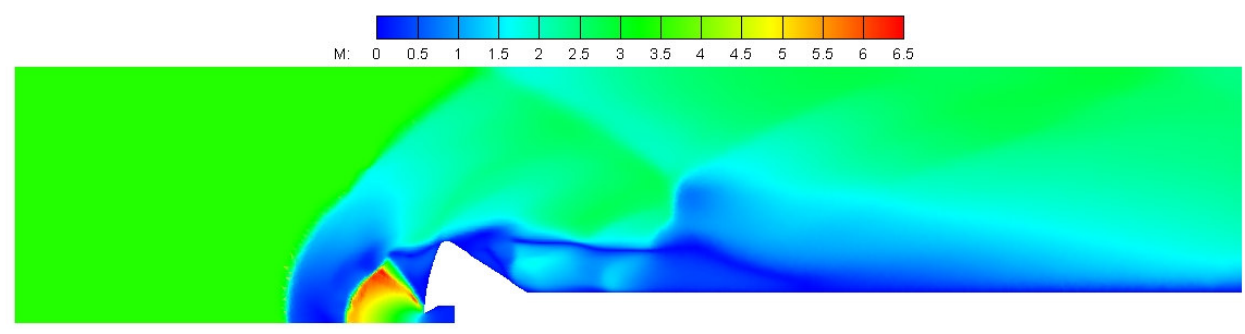

(b) Mach number
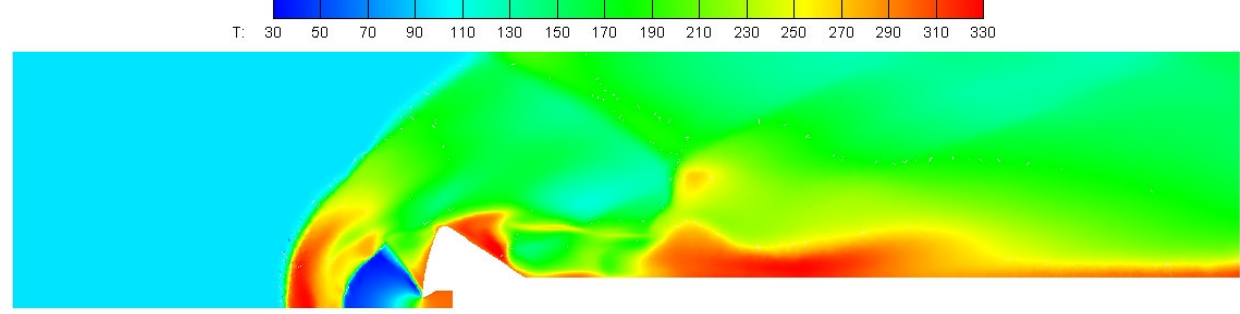

(c) Temperature (K)

Figure 11: Computed flowfield at the symmetry plane for Case $2\left(M_{.}=3.48\right.$, counterflow jet exit Mach number of $1.0)$.

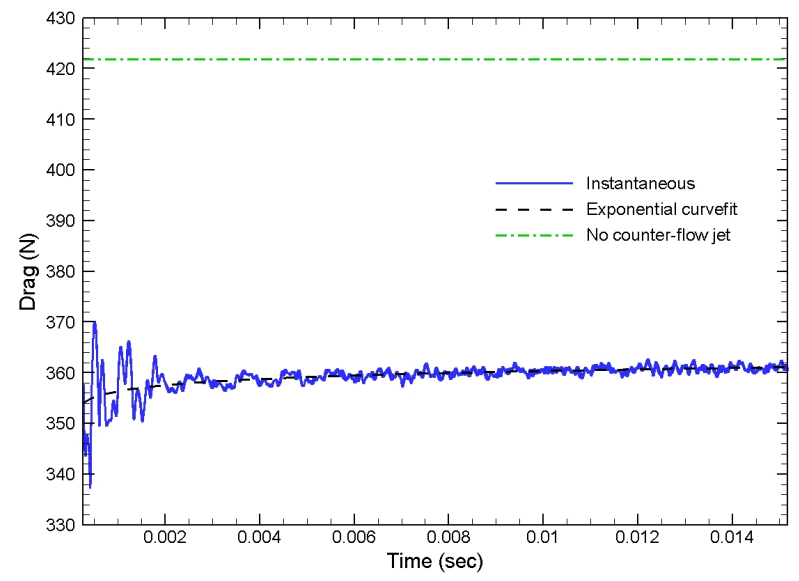

Figure 12: Time history of the drag force on the face of the capsule for Case $2(\mathrm{M} .=3.48$, counterflow jet exit Mach number of 1.0).

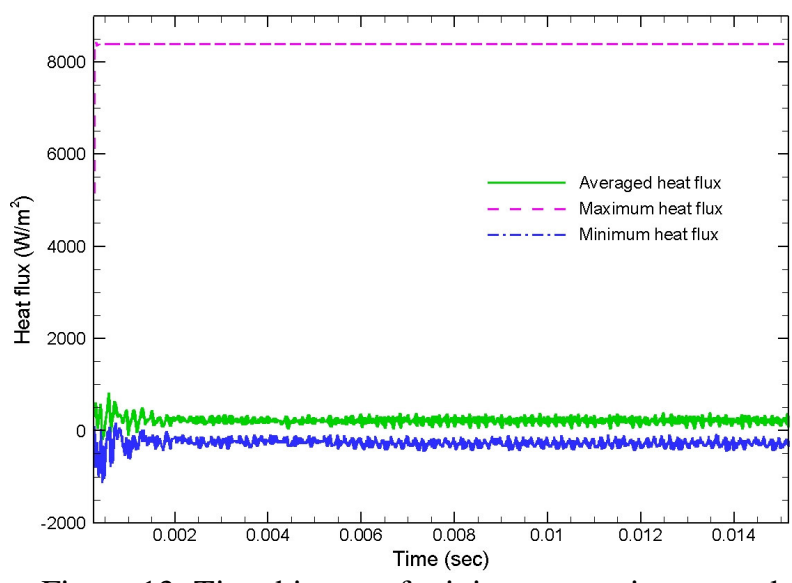

Figure 13: Time history of minimum, maximum, and averaged heat fluxes to the capsule face for Case 2 (M. = 3.48, counterflow jet exit Mach number of 1.0). 


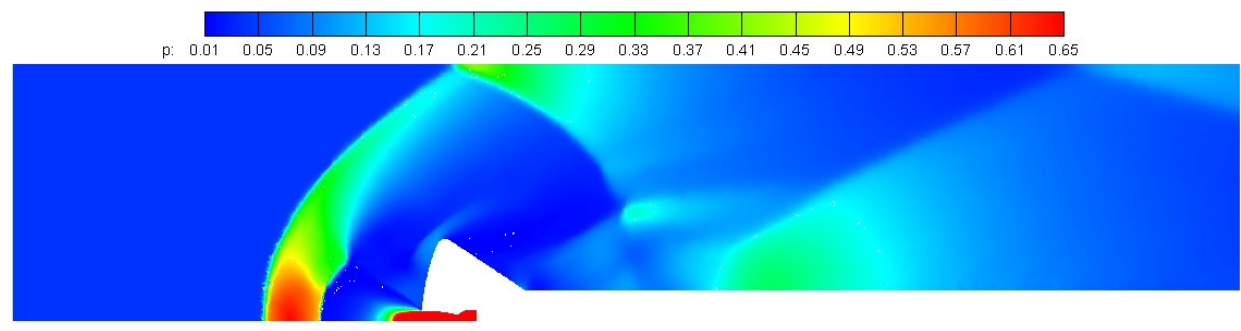

(a) Pressure (atm)

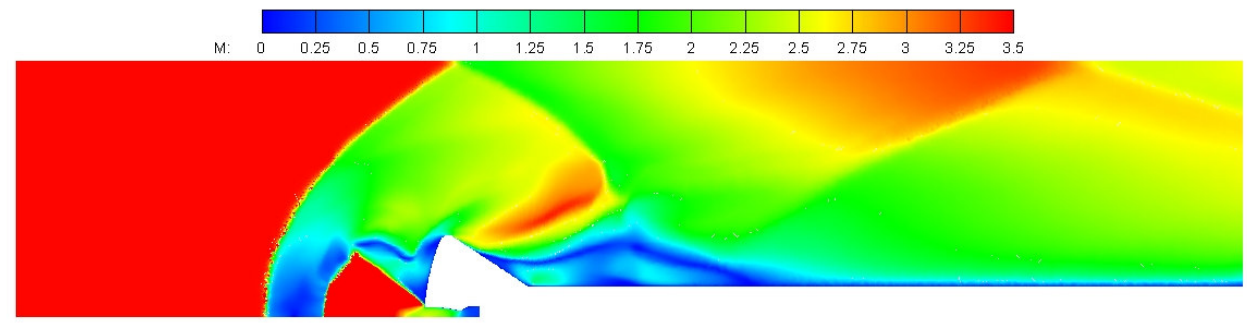

(b) Mach number

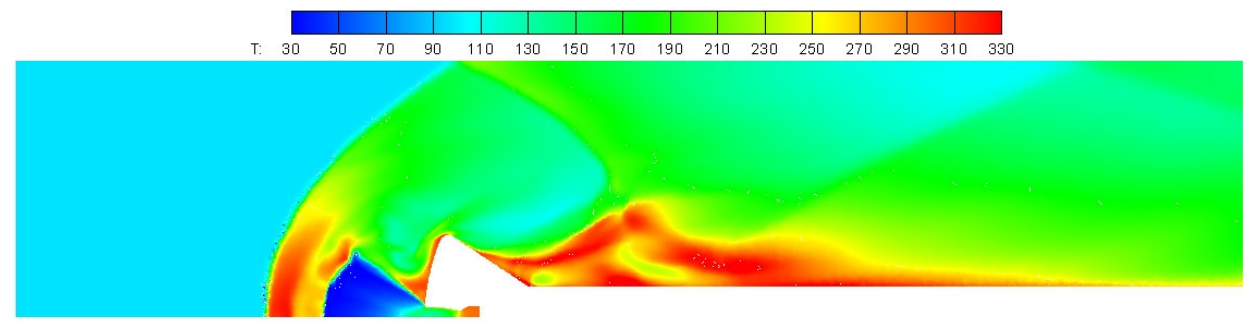

(c) Temperature (K)

Figure 14: Computed flowfield at the symmetry plane for Case $3(\mathrm{M} .=3.48$, counterflow jet exit Mach number of 2.44).

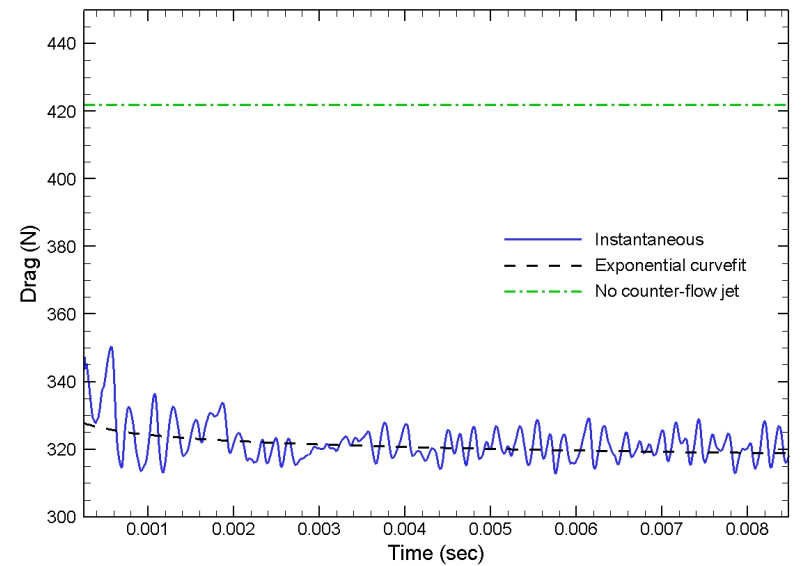

Figure 15: Time history of the drag force on the face of the capsule for Case $3(\mathrm{M} .=3.48$, counterflow jet exit Mach number of 2.44).

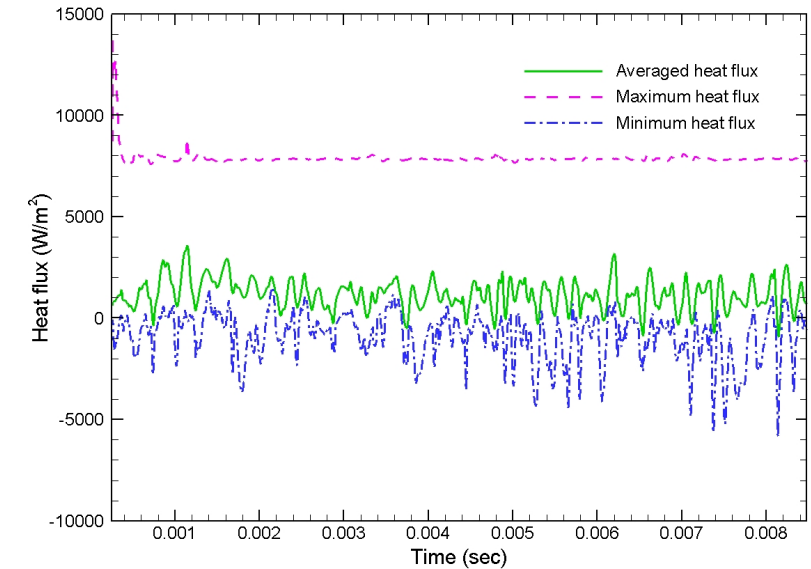

Figure 16: Time history of minimum, maximum, and averaged heat fluxes to the capsule face for Case 3 (M. = 3.48, counterflow jet exit Mach number of 2.44). 


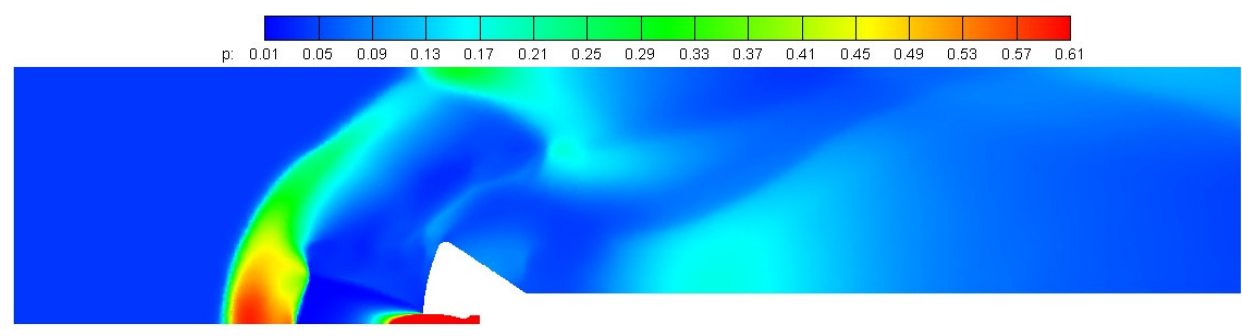

(a) Pressure (atm)

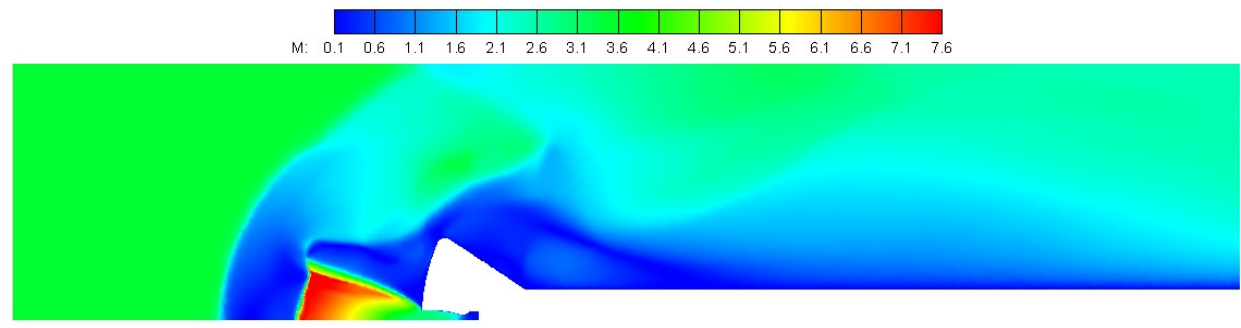

(b) Mach number

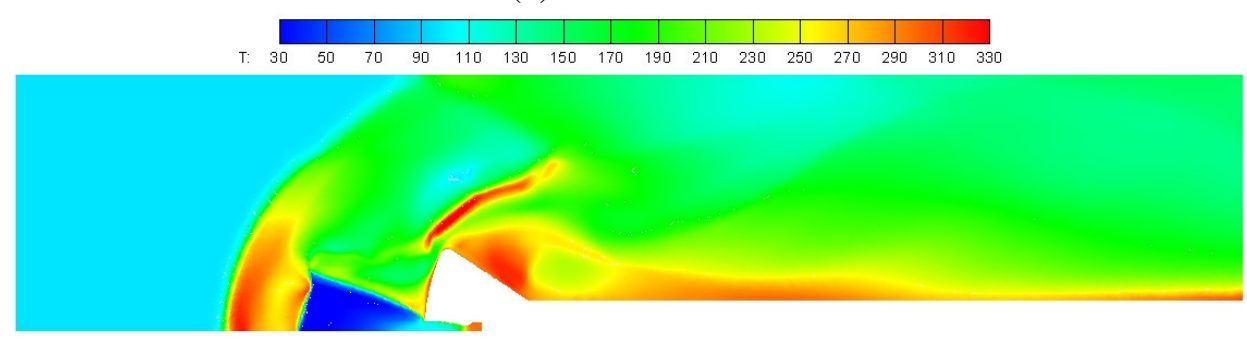

(c) Temperature (K)

Figure 17: Computed flowfield at the symmetry plane for Case $4(M .=3.48$, counterflow jet exit Mach number of 2.94).

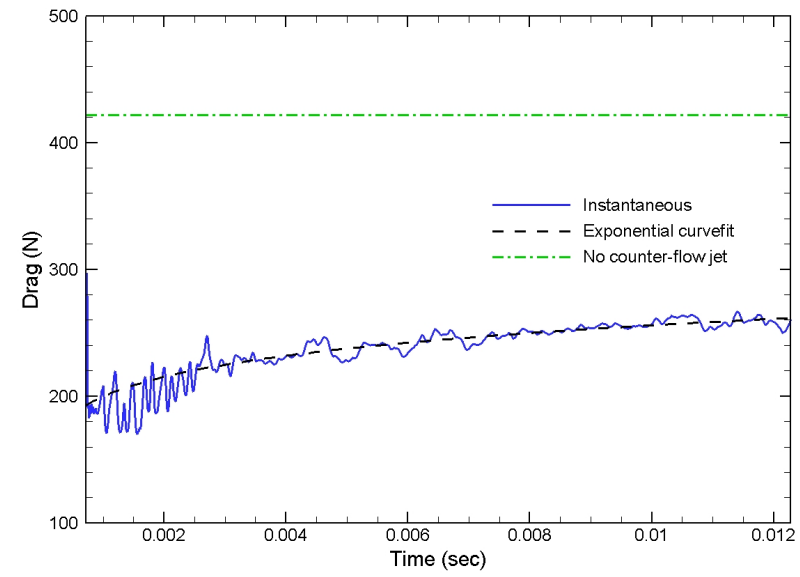

Figure 18: Time history of the drag force on the face of the capsule for Case $4(\mathrm{M} .=3.48$, counterflow jet exit Mach number of 2.94).

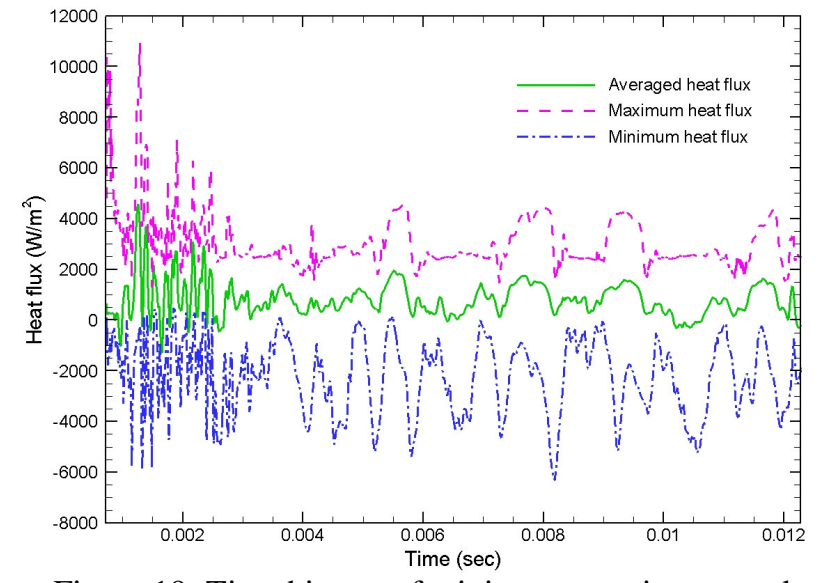

Figure 19: Time history of minimum, maximum, and averaged heat fluxes to the capsule face for Case 4 (M. = 3.48, counterflow jet exit Mach number of 2.94). 


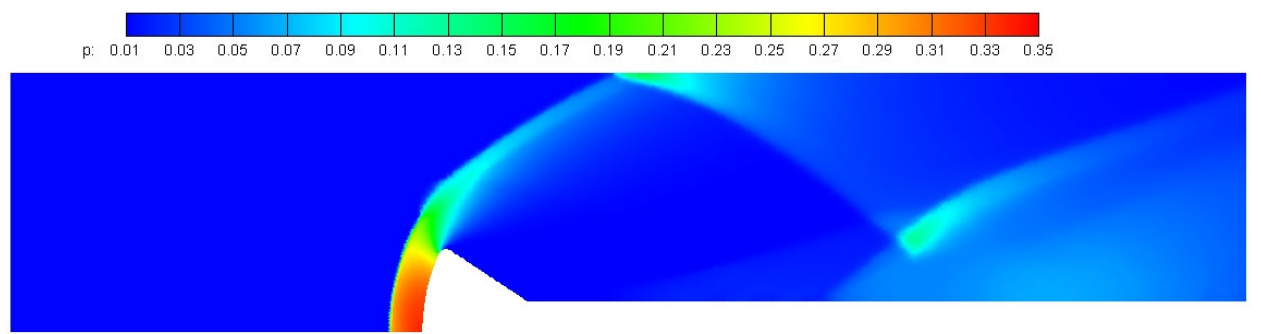

(a) Pressure (atm)

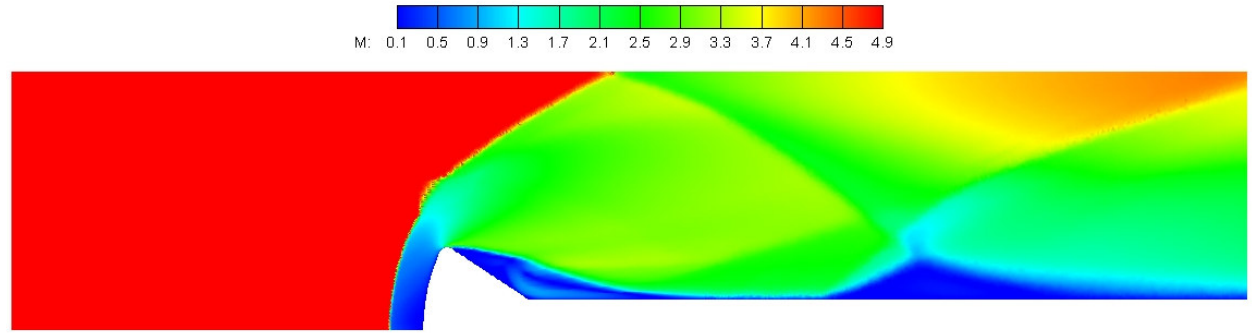

(b) Mach number

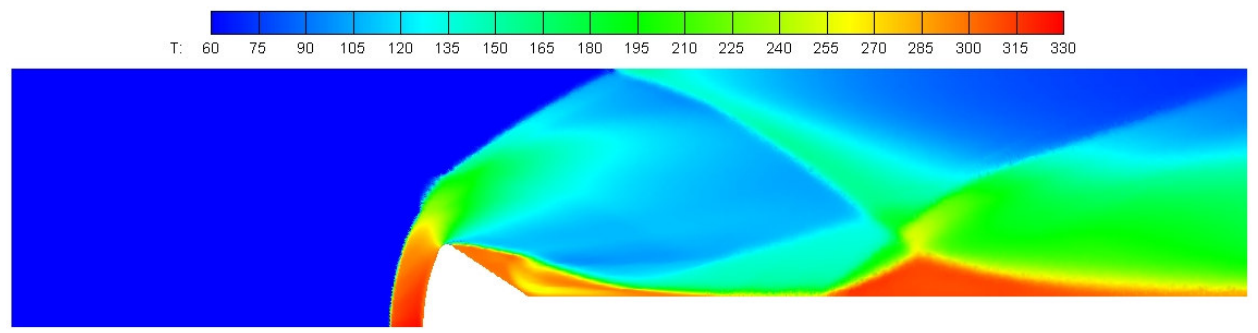

(c) Temperature (K)

Figure 20: Computed flowfield at the symmetry plane $(M=4.96$, no jet $)$.

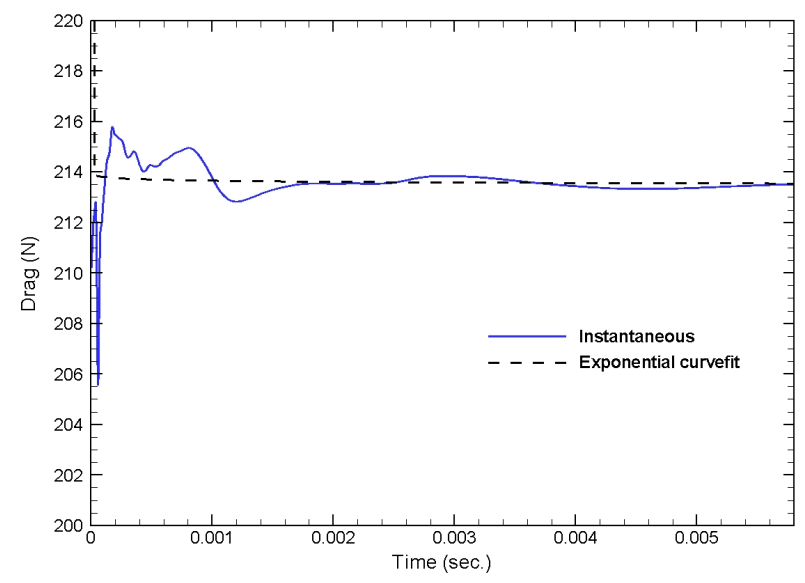

Figure 21: Time history of the drag force on the face of the capsule ( $M=4.96$, no counterflow jet).

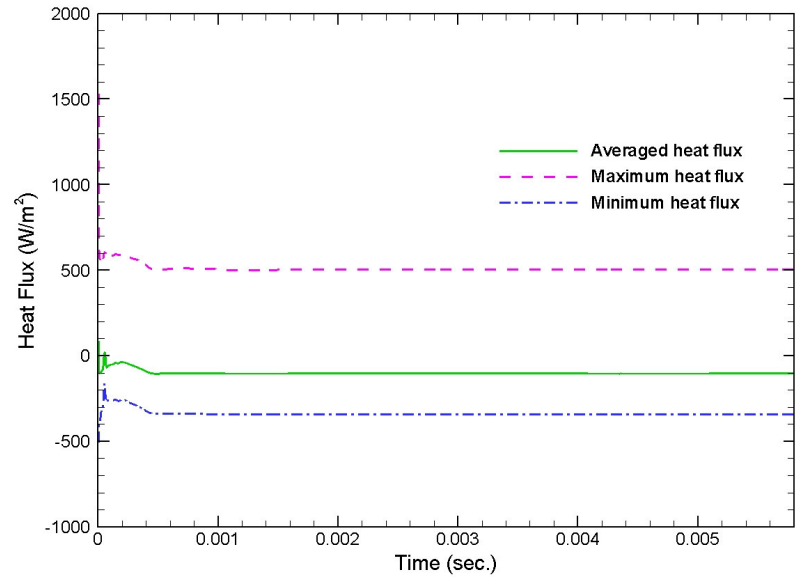

Figure 22: Time history of minimum, maximum, and averaged heat fluxes to the capsule face $(M .=4.96$, no counterflow jet). 


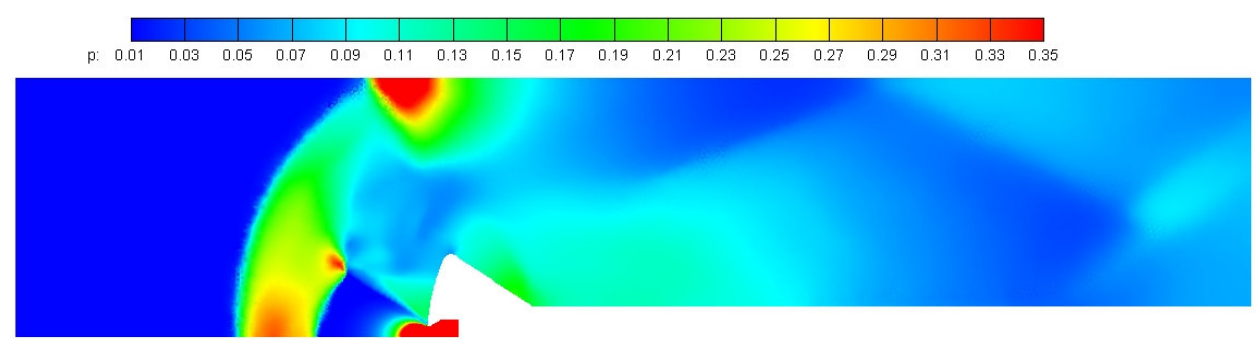

(a) Pressure (atm)

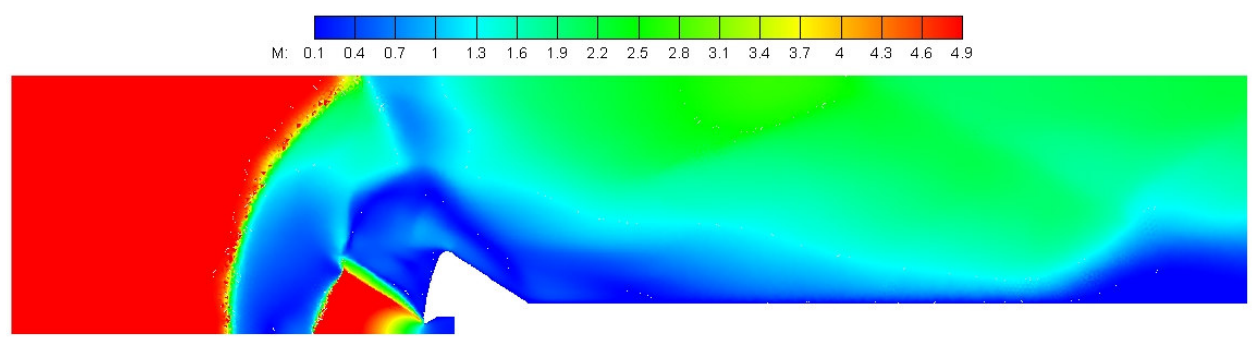

(b) Mach number

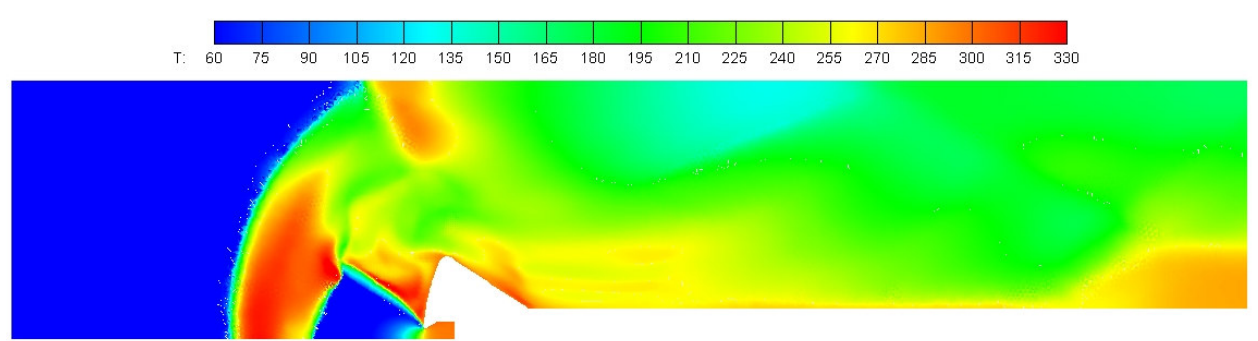

(c) Temperature $(\mathrm{K})$

Figure 23: Computed flowfield at the symmetry plane $(M=4.96$, counterflow jet exit Mach number of 1)

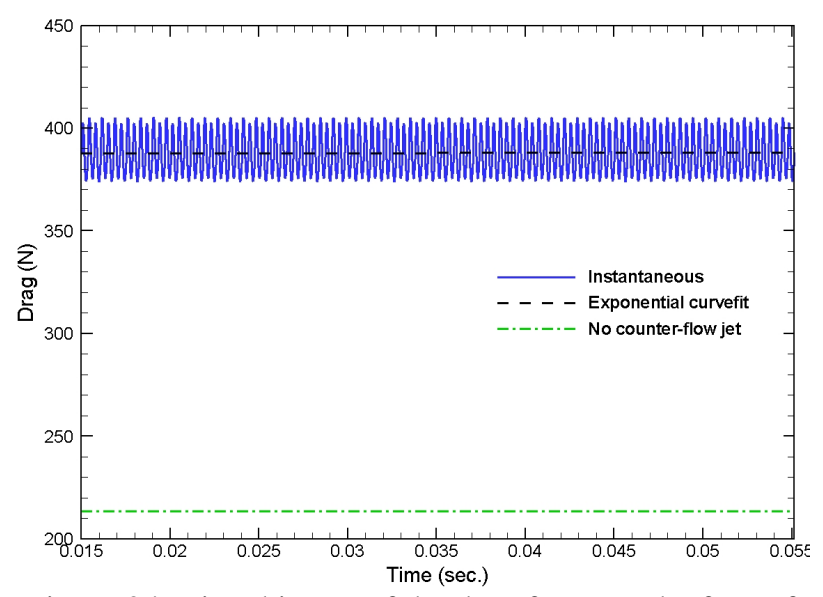

Figure 24: Time history of the drag force on the face of the capsule $(\mathrm{M}=4.96$, Angle counterflow jet exit Mach number of 1)

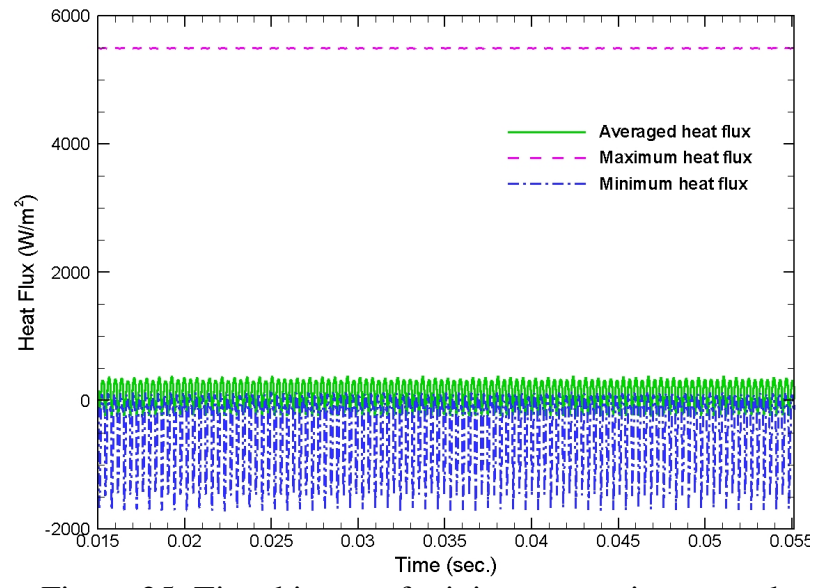

Figure 25: Time history of minimum, maximum, and averaged heat fluxes to the capsule face $(M=4.96$, counterflow jet exit Mach number of 1) 


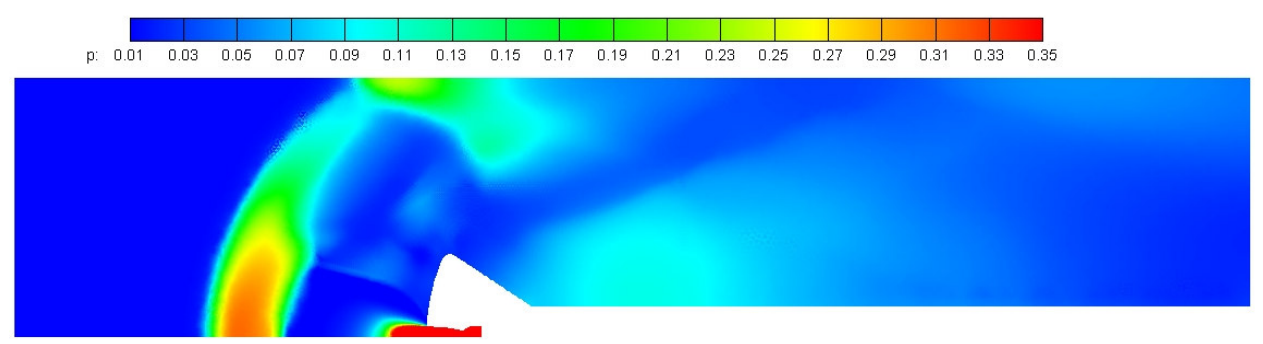

(a) Pressure (atm)

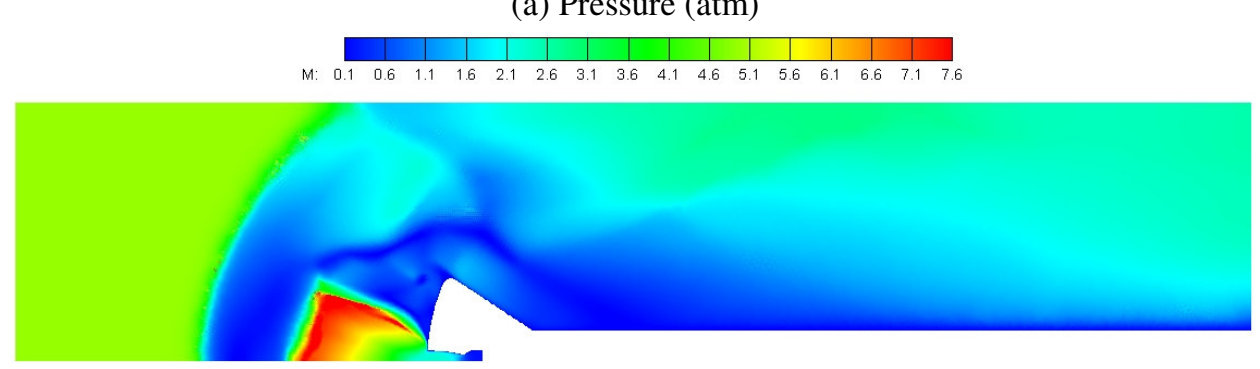

(b) Mach number

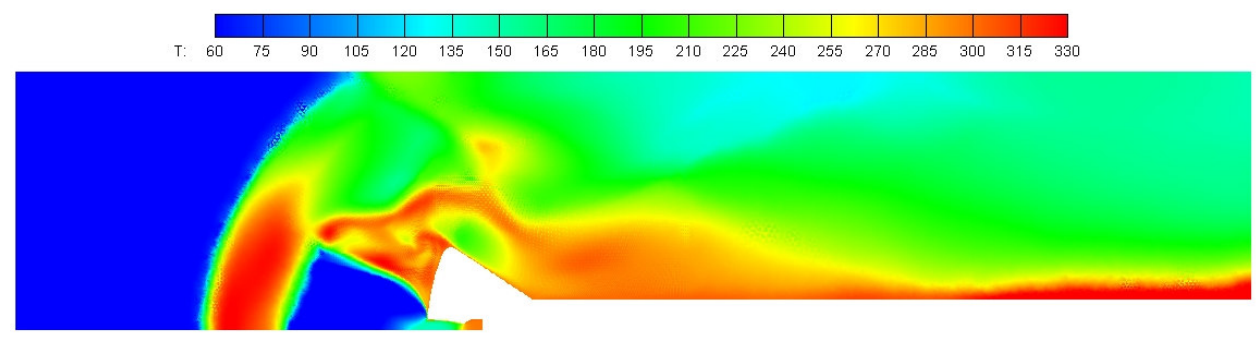

(c) Temperature $(\mathrm{K})$

Figure 26: Computed flowfield at the symmetry plane ( $M=4.96$, counterflow jet exit Mach number of 2.44)

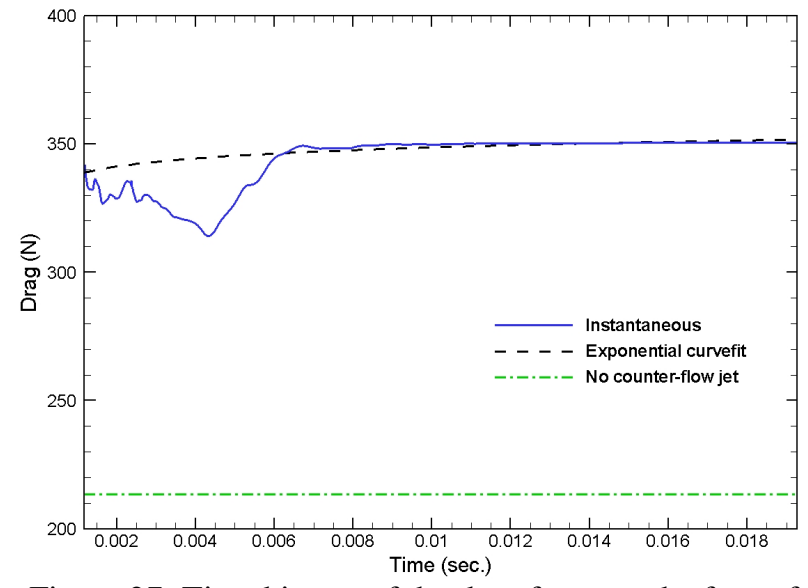

Figure 27: Time history of the drag force on the face of the capsule $(M=4.96$, counterflow jet exit Mach number of 2.44)

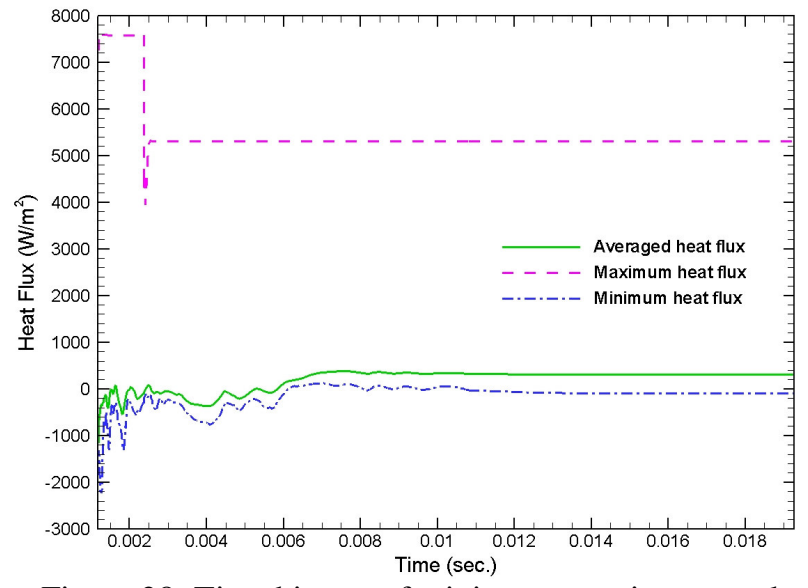

Figure 28: Time history of minimum, maximum, and averaged heat fluxes to the capsule face $(M=4.96$, counterflow jet exit Mach number of 2.44) 


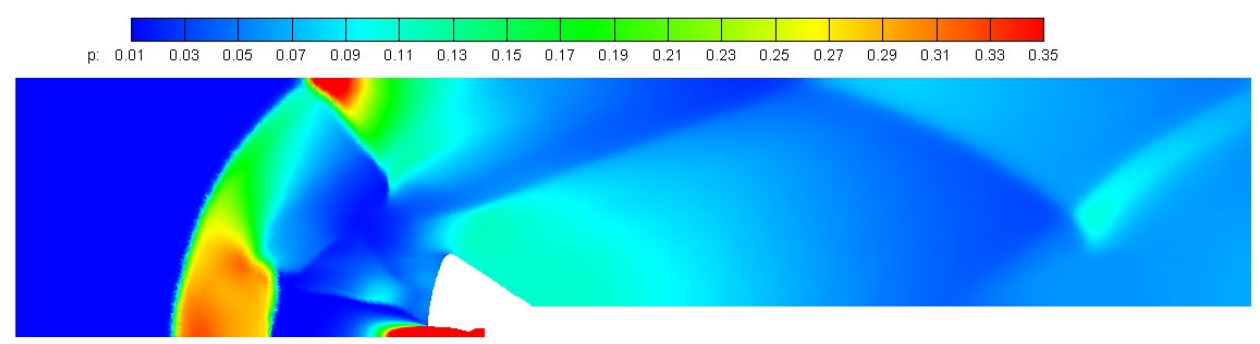

(a) Pressure (atm)

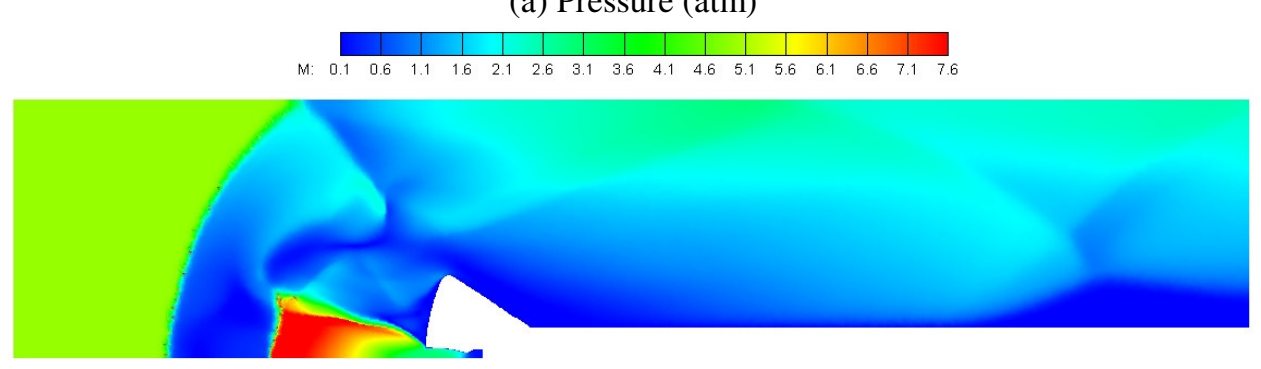

(b) Mach number

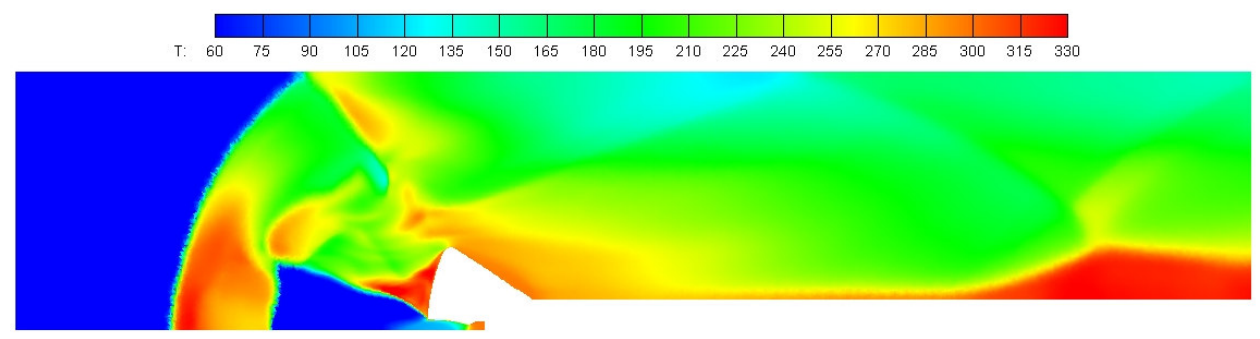

(c) Temperature $(\mathrm{K})$

Figure 29: Computed flowfield at the symmetry plane ( $M=4.96$, counterflow jet exit Mach number of 2.94)

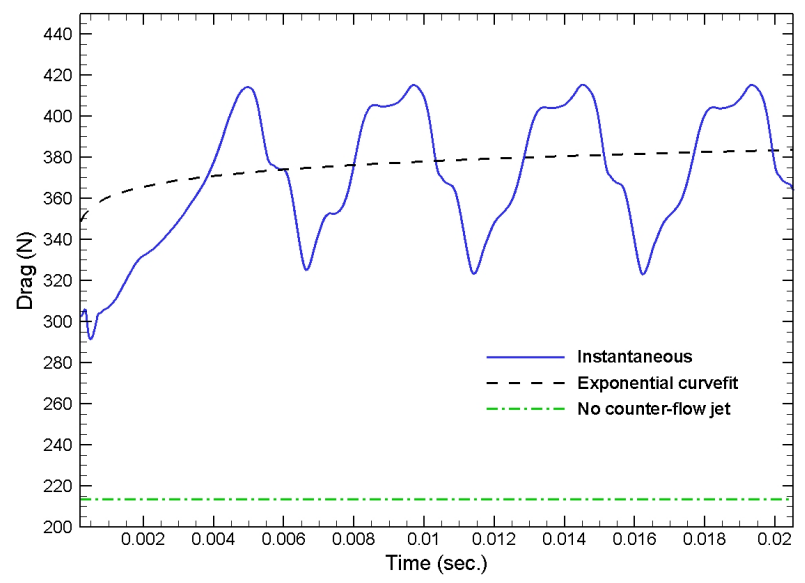

Figure 30: Time history of the drag force on the face of the capsule $(M=4.96$, counterflow jet exit Mach number of 2.94)

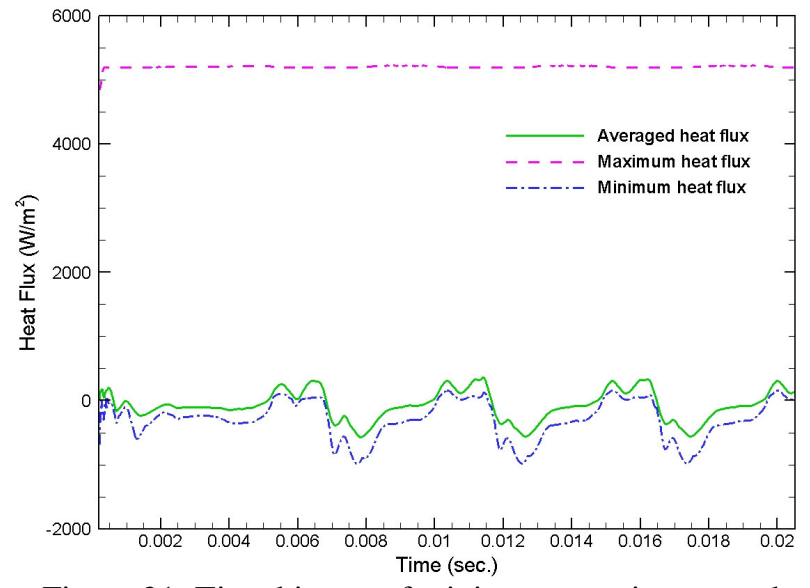

Figure 31: Time history of minimum, maximum, and averaged heat fluxes to the capsule face $(M=4.96$, counterflow jet exit Mach number of 2.94) 


\section{Summary}

The space-time CESE method is used to numerically investigate supersonic flows over a capsule with and without a counterflow jet. The counterflow jet is intended for better thermal management of the entry or reentry capsules. A set of capsule/jet configurations under two different wind tunnel conditions from an on-going experimental work [10] are calculated. The computed flowfield is shown to compare well with experimental Schlieren pictures. The counterflow jet results in a significant increase of the bow shock stand-off distance, accompanied by strong flow unsteadiness. Among all cases, the smaller free stream Mach number $(\mathrm{M}=3.8)$ tunnel cases result in a significant drag reduction due to the presence of the counterflow jet. The amount of drag reduction is comparable to that previously reported in the literature. On the contrary, the higher Mach tunnel cases $(M=4.96)$ show a substantial increase in drag when a counterflow jet is present. The capsule surface heat flux varies over a large range. Due to a low model wall temperature, the average heat flux on the capsule face increases due to the counterflow jet. More appropriate thermal environment is necessary to assess the net benefit of a counterflow jet on heat transfer to the capsule face. The results documented in this study can be used for validation of wind tunnel data and they also provide more insights into the flow physics involved in a capsule configuration with or without a supersonic counterflow jet.

\section{Acknowledgments}

The authors would like to thank Drs. Daso and Pritchett of NASA Marshall Space Flight Center for providing unpublished experimental Schlieren photos and other details of the counterflow jet capsule experiments. Valuable comments suggested by Dr. T.S. Wang of NASA Marshall Space Flight Center are greatly appreciated.

\section{References}

1. http://www.nasa.gov/mission_pages/exploration/spacecraft/work_assign.html

2. Jones, R., "Experimental Investigation of the Overall Pressure Distribution, Flow Field, and Afterbody Heat Transfer Distribution of an Apollo Reentry Configuration at a Mach Number of 8," NASA TM X813, Jun. 1963.

3. Bertin, J., "Wind Tunnel Heating Rates for the Apollo Spacecraft," NASA TM X-1033, Jan. 1965.

4. Lee, D.B., Bertin, J.J., and Goodrich, W.D., "Heat Transfer Rate and Pressure Measurements Obtained During Apollo Orbital Entries," NASA TN D-6028, Oct. 1970.

5. Lee, D.B. and Goodrich, W.D., "The Aerothermodynamic Environment of the Apollo Command Module During Superorbital Entry,” NASA TN D-6792, Apr. 1972.

6. Lee, D.B, “Apollo Experience Report: Aerothermodynamics Evaluation,” NASA TN D-6843, Jun. 1972.

7. Wright, M. J., Prabhu, D. K., and Martinez, E. R., "Analysis of Afterbody Heating Rates on the Apollo Command Modules, Part 1: AS202,” AIAA Paper 2004-2456, 2004.

8. Lee, G. and Sundell, R. E., "Apollo Afterbody Heat Transfer and Pressure with and without Ablation at $\mathrm{M}_{\infty}$ of 5.8 to 8.3," NASA TN D-3620, September, 1966.

9. Park, C. and Tauber, M. E., "Heatshielding Problems of Planetary Entry, A Review," AIAA Paper 993415, 1999.

10. Daso, E. O. and Pritchett, "Flow Augmented Thermal Management in Entry and Re-Entry Environments," NASA Langley Research Center Internal Presentations, March 29th, 2006.

11. Romeo, D. and Sterrett, J., "Exploratory Investigation of the Effect of a Forward Facing Jet on the Bow Shock of a Blunt Body in a Mach Number 6 Free Stream," Tech. Rep. NASA TN D-1605, 1963.

12. Shan Finley, P. J., "The Flow of a Jet from a Body Opposing a Supersonic Free Stream," J. Fluid Mech., Vol. 26, Part 2, pp. 337-368, 1966.

13. J. S., Hayes, J., and Wurtzler, K., "Jet-Spike Bifurcation in High-Speed Flows," AIAA Paper 2000-2325, 2000. 
14. Joshula, E., Pinney, M., and Blake, W. B., "Applications of a Counterflow Drag Reduction Technique in High Speed Systems," AIAA Paper 2001-2437, 2001.

15. Pandolfi, M. and Ambrosio, D. D., "Numerical Instabilities in Upwind Methods: Analysis and Cures for the "Carbuncle" Phenomenon," J. Comput. Physics, Vo. 166, pp. 271-301, 2001.

16. Robinet, J.-Ch., Gressier, J., Casalis, G., and J.-M. Moschetta, "Shock Wave Instability and the Carbuncle Phenomenon: Same Intrinsic Origin?” J. Fluid Mech. Vo. 417, pp. 237-263, 2000.

17. Chang, S.-C., "The Method of Space-Time Conservation Element and Solution Element - A New Approach for Solving the Navier-Stokes can Euler Equations," J. Comput. Physics, Vol. 119, pp.295-324, 1995.

18. Venkatachari, B. S. and Cheng, G. C., and Chang, S.-C., "Courant Number Insensitive Transient Viscous Flow Solver Based on CE/SE Framework," AIAA Paper 2005-0093, 2005.

19. Chang, C.-L., "Time-Accurate, Unstructured-Mesh Navier-Stokes Computations with the Space-Time CESE Method," AIAA Paper 2006-4780, 2006.

20. Zhang, M., S.-C. Chang, H. Lin, S. T. J. Yu, "Application of the Space-Time CE/SE Method to the Ideal Magnetohydrodynamic Equations," AIAA paper 2002-3888, 2002.

21. Loh, C., Hultgren, L. S., and Chang, S.-C., "Wave Computation in Compressible Flow Using Space-Time Conservation Element and Solution Element Method," AIAA J. Vol. 39, No. 5, pp. 794-801, 2001.

22. Chang, I.-S., Chang, C.-L., and Chang, S.-C., "Unsteady Navier-Stokes Rocket Nozzle Flows," AIAA Paper 2005-4353, 2005.

23. Chang, S.-C. and Wang, X.-Y., "Multi-Dimensional Courant Number Insensitive CE/SE Euler Solvers for Applications Involving Highly Non-uniform Meshes," AIAA Paper 2003-5285, 2003.

24. Chen, Y.S., "Compressible and Incompressible Flow Computations with a Pressure Based Method," AIAA Paper 89-0286

25. Wang, T.S., and Chen, Y.S., "Unified Navier-Stokes Flowfield and Performance Analysis of Liquid Rocket Engines," Journal of Propulsion and Power, Vol. 9, pp. 678-685, 1993. 